\title{
A Hybrid Automata Approach for Monitoring the Patient in the Loop in Artificial Pancreas Systems
}

\author{
Aleix Beneyto $^{1}\left(\mathbb{D}\right.$, Vicenç Puig ${ }^{2}\left(\mathbb{D}\right.$, B. Wayne Bequette $^{3}(\mathbb{C})$ and Josep Vehi ${ }^{1,4, *(1)}$ \\ 1 Department of Electrical, Electronic and Automatic Engineering, University of Girona, 17004 Girona, Spain; \\ aleix.beneyto@udg.edu \\ 2 Automatic Control Department-Campus de Terrassa, Universitat Politècnica de Catalunya (UPC), \\ 08222 Terrassa, Spain; vicenc.puig@upc.edu \\ 3 Department of Chemical Engineering, Rensselaer Polytechnic Institute, Troy, NY 12180, USA; \\ bequette@rpi.edu \\ 4 Centro de Investigación Biomédica en Red de Diabetes y Enfermedades Metabólicas Asociadas, \\ 28029 Madrid, Spain \\ * Correspondence: josep.vehi@udg.edu
}

check for updates

Citation: Beneyto, A.; Puig, V.; Bequette, B.W.; Vehi, J. A Hybrid Automata Approach for Monitoring the Patient in the Loop in Artificial Pancreas Systems. Sensors 2021, 21, 7117. https://doi.org/10.3390/ s21217117

Academic Editor: Jonathan Golledge

Received: 2 September 2021

Accepted: 23 October 2021

Published: 27 October 2021

Publisher's Note: MDPI stays neutral with regard to jurisdictional claims in published maps and institutional affiliations.

Copyright: (c) 2021 by the authors. Licensee MDPI, Basel, Switzerland. This article is an open access article distributed under the terms and conditions of the Creative Commons Attribution (CC BY) license (https:/ / creativecommons.org/licenses/by/ $4.0 /)$.

\begin{abstract}
The use of automated insulin delivery systems has become a reality for people with type 1 diabetes (T1D), with several hybrid systems already on the market. One of the particularities of this technology is that the patient is in the loop. People with T1D are the plant to control and also a plant operator, because they may have to provide information to the control loop. The most immediate information provided by patients that affects performance and safety are the announcement of meals and exercise. Therefore, to ensure safety and performance, the human factor impact needs to be addressed by designing fault monitoring strategies. In this paper, a monitoring system is developed to diagnose potential patient modes and faults. The monitoring system is based on the residual generation of a bank of observers. To that aim, a linear parameter varying (LPV) polytopic representation of the system is adopted and a bank of Kalman filters is designed using linear matrix inequalities (LMI). The system uncertainty is propagated using a zonotopic-set representation, which allows determining confidence bounds for each of the observer outputs and residuals. For the detection of modes, a hybrid automaton model is generated and diagnosis is performed by interpreting the events and transitions within the automaton. The developed system is tested in simulation, showing the potential benefits of using the proposed approach for artificial pancreas systems.
\end{abstract}

Keywords: artificial pancreas; hybrid automaton; Kalman filter; patient in the loop; type 1 diabetes

\section{Introduction}

Type 1 diabetes (T1D) is a serious metabolic disease characterized by an autoimmune destruction of the insulin-producing $\beta$-cells in the pancreas and subsequent insulin deficiency. Insulin is a hormone that allows glucose uptake from the blood into cells, either to be used as fuel or stored for future use. Low levels of insulin inevitably lead to high blood glucose (BG) concentrations, known as hyperglycemia, which can also lead to long-term complications $[1,2]$. Current therapies are based on administering exogenous insulin using devices such as insulin pumps or pens [3,4].

Artificial pancreas (AP) is a closed-loop (CL) system in which insulin is delivered automatically by adjusting a pump's insulin infusion rate depending on continuous glucose monitor (CGM) readings [5]. CGM sensors measure glucose subcutaneously and provide an estimate of current BG levels. Many different control strategies for AP are available in the literature [5-7]. AP systems have been an important focus of research and discussion over the last years, with a multitude of clinical trials being conducted world-wide [8]. There is clinical evidence that suggests that using an AP is safe, robust, and efficacious for people with T1D when compared to traditional open-loop therapy $[7,8]$. 
However, AP technology is not exempt of risks during operation [9]. It is especially critical to take into account the concept of patient-in-the-loop in the design phase for this kind of technology [10], because ultimately, patients will wear and operate APs. Patients will have to provide inputs, maintain the system, and ideally undertake training before using any AP. Therefore, the patient will play a significant role in any configuration-a clear example is the Medtronic MiniMed 670G AP [11]. Undoubtedly, research is trying to find solutions that reduce the impact of patient behaviors and decisions. Therefore, any developed AP must implement fault tolerant control (FTC) strategies to mitigate faults and ensure and maintain stability, performance, and safety for the patient.

In the context of FTC research for AP systems, most of the literature is focused on fault detection (FD) and fault identification (FI) for either the CGM [12,13] or insulin pumps [14-17]. Only few tackle the issue of how the patient-in-the-loop affects the system and what potential faults may arise from it [9]. Developed strategies to detect specific patient behaviors exist, however, they were not developed for safety but as ad-hoc approaches for control. Most of the approaches involving the patient estimate the glucose rate of appearance to allow AP to operate without meal announcement [18-23]. Other strategies try to detect when patients exercise and modify or adapt their control strategy accordingly [24,25]. In any case, the roads being followed are either hybrid systems, which rely on patient input, or fully CL systems. The patient-in-the-loop paradigm links both approaches allowing fully CL systems to operate, but also to rely on contrasted patient input information to enhance performance and safety. Hence, patient modes and inputs should be monitored and potential faults must be uncovered for fully CL systems that may from time to time may use patient information. Here, a patient operational mode is defined by its significantly different system dynamics, for example the increased insulin sensitivity during aerobic exercise. Additionally, patient inputs are considered as any action that the patient can perform with direct implications to BG control.

The aim of this work is to provide robust and safe solutions to deal with the patientin-the-loop in AP systems. In the context of diabetes and AP systems, the patient should be considered as part of the control loop [26]. Depending on the system configuration, the patient may have to take the role of an actuator or a sensor, besides being the plant to control. Specifically, the subjects will be considered actuators performing the control action of eating the recommended carbohydrates (CHO) [27] or informing about meal consumption or exercise. It is known that patient information can be erroneous, for example, studies show that patients have significant estimation errors when counting $\mathrm{CHO}$ [28]. Therefore, the detection and monitoring of patient modes can be used to help the control system in maintaining performance and safety despite patient faults. Considering the patient-in-the-loop, the monitoring of system modes can be aided by the feed-forward information provided by the patient. That information is inherently affected by uncertainty and must be checked to exclude any possible fault.

The implemented approach is a model-based FD system and can work with any kind of hybrid or fully CL control architecture that announces meals and/or uses insulin and/or $\mathrm{CHO}$ as a control action. The physiology of a person with T1D is subjected to highly nonlinear phenomena. A way of extending traditional linear system theory to nonlinear systems is by means of the linear varying parameter (LPV) paradigm [29]. Here, this approach will be used for modeling T1D subjects. Then, we design a bank of polytopic Kalman filters by solving the dual of the linear quadratic control (LQC) problem and imposing constraints on the system stability and performance by using linear matrix inequalities (LMI). Dealing with system uncertainties in model-based approaches is known to be an important factor. In this work, we use a deterministic approach using zonotopes to build interval observers (IO). The resulting bank of IO will be used to generate a set of residual signals online. A hybrid automaton (HA) is built based on a configuration of normal and faulty patient modes. Transitions between modes are done by analyzing the patient input events and checking the residuals consistency. 
This paper is organized as follows: Section 2 presents the problem formulation and briefly describes the control system. Section 3 describes the patient monitoring system. Section 4 describes the modeling process of a T1D patient. Section 5 is focused on the residual generation by designing IO. In Section 6, in-silico benchmarks are proposed to evaluate the monitoring system. Finally, Sections 7 and 8 include the discussion and conclusions from this work.

\section{Problem Statement}

The Spanish consortium on artificial pancreas and diabetes technology (eSCAPE) has developed a multivariable hybrid AP system for the regulation of glucose to cope with the two disturbances that have the biggest effect on BG, meals, and exercise. The system uses both insulin and $\mathrm{CHO}$ as control actions to maintain BG in the euglycemic range $70-140 \mathrm{mg} / \mathrm{dL}$. The insulin feedback loop is based on a proportional-derivative (PD) controller with insulin feedback (IFB) that integrates a safety layer with insulin-on-board (IOB) constraints and sliding mode reference conditioning (SMRC) [30,31]. The second feedback loop uses a predictive PD controller with a quantization system that encourages the patient to consume $\mathrm{CHO}$ if there is danger of hypoglycemia [27]. Both control loops are coordinated by using coupled carbohydrates-on-board (COB) inhibition signals that ensure that insulin and $\mathrm{CHO}$ control actions are effective and not mutually counteracted.

The system has recently been upgraded with additional modules to enhance performance and safety when exercise is announced. If patients announce exercise, a feed-forward controller will adapt the insulin gains and, if required, the consumption of a snack will be suggested [32]. Furthermore, we included an adaptive IOB system to enhance postprandial control [33]. This control system has already been tested in clinical trials against meals and exercise and has showed promising results [34-36]. The overall control strategy is depicted in Figure 1 and is the control system that will be used in this work.

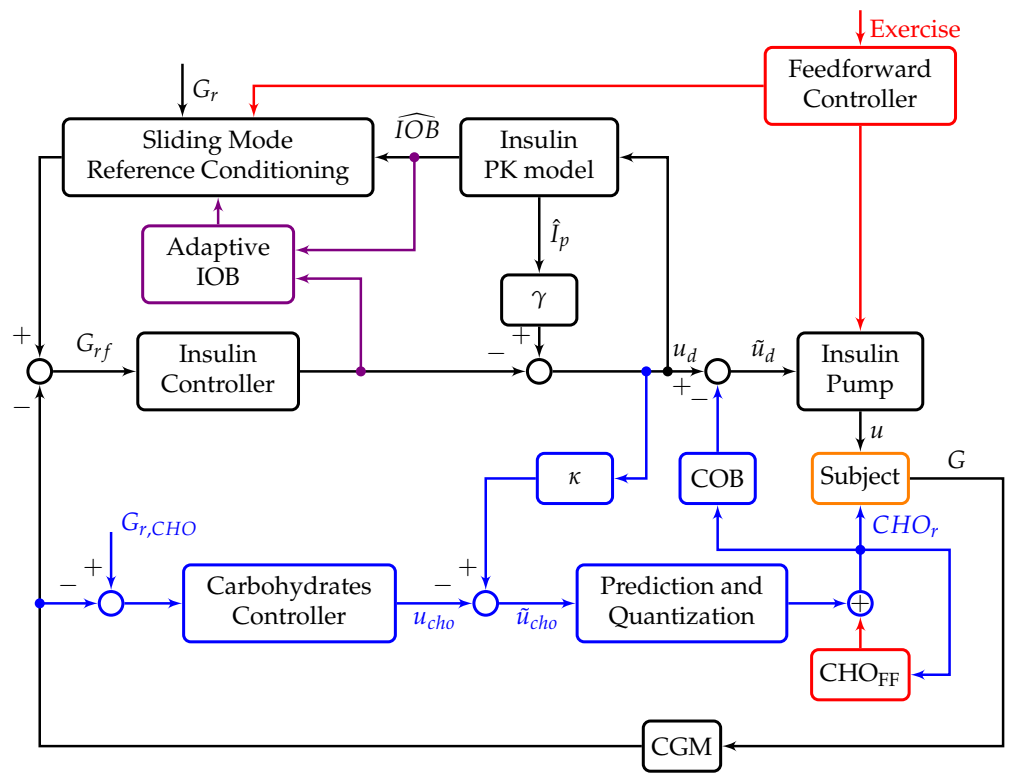

Figure 1. Multivariable hybrid AP. The black blocks and signals are elements from the insulin feedback loop, the blue from the CHO feedback loop, the red from the feed-forward exercise control, the violet is the IOB adaptive algorithm and the orange block is the patient to control.

This system is a hybrid AP where the patient takes fundamental roles. We want to address the patient-in-the-loop situation that arises when using patients as an operator and as the plant to control. In this configuration, patients play a sensor role when announcing meals and exercise. They also play an actuator role when the $\mathrm{CHO}$ controller suggests rescue $\mathrm{CHO}$. Therefore, patients can introduce errors into the system that could eventually lead to faults. We consider patient-in-the-loop faults as poorly estimated meal boluses, 
when patients do not follow the $\mathrm{CHO}$ controller recommendations, or when the dynamical plant is behaving in an unexpected manner.

\section{Materials and Methods}

In this work, we develop a system for the detection of patient modes by using the scheme proposed in [37] based on creating a HA model that presents as many states as patient modes. For mode detection, we will generate a set of residual signals. Checking at every time instant which residuals are consistent with the current mode, we can detect the change of mode. Residuals will be generated by using a bank of zonotopic Kalman filters.

\subsection{Hybrid Automaton}

The HA has been developed based on [37]. The idea behind using an HA is to mimic real patient operational modes and transitions. Using this approach, the HA is defined by the following components, $H A^{k}=\langle\mathcal{Q}, \mathcal{X}, \mathcal{U}, \mathcal{Y}, \mathcal{F}, \mathcal{G}, \mathcal{H}, \Sigma, \mathcal{T}\rangle$ where:

1. $\mathcal{Q}$ is the set of modes. Each of the modes $q_{i} \in \mathcal{Q}$ represents an operational mode of the patient. The set of modes is constituted by nominal and faulty operational modes, i.e., $\mathcal{Q}=\mathcal{Q}_{N} \cup \mathcal{Q}_{F}$. Particularly, we consider three nominal operational modes and three faulty modes, see Figure 2;

2. $\mathcal{X} \subseteq \mathbb{R}^{n_{x}}$ defines the state space of the system on each of the modes $\mathbf{x}(k) \in \mathcal{X}$, where $\mathbf{x}(k)$ is the state space vector. $\mathcal{U} \subseteq \mathbb{R}^{n_{u}}$ and $\mathcal{Y} \subseteq \mathbb{R}^{n_{y}}$ define the continuous input and output spaces. In this work, the input space includes both insulin, rescue $\mathrm{CHO}$ and meal inputs, and the output space only considers the CGM measurements. $\mathcal{G}$ defines the set of discrete time state functions and $\mathcal{H}$ is the set of discrete time output functions;

3. $\Sigma=\Sigma_{S} \cup \Sigma_{C} \cup \Sigma_{\mathcal{F}}$ is a set of events. This set of events can be split into spontaneous mode switching events $\Sigma_{S}$, input events $\Sigma_{C}$ and fault events $\Sigma_{\mathcal{F}}$. Spontaneous switching events are unknown events that may produce transitions between modes in the real patient. For example, an unannounced meal may lead to a spontaneous transition to the meal operational mode, even if that event (eating) is unknown to the system. Input events are defined by patient actions, such as announcing meals or exercise. Fault events are defined by checking the consistency of the residuals and the ending transitioning mode;

4. $\mathcal{F}$ is the set of possible faults. For each faulty mode $q_{i} \in \mathcal{Q}_{F}$ the system has a specific $f_{i} \in \mathcal{F}$, which is associated with a fault event $\Sigma_{F}$;

5. $\mathcal{T}: \mathcal{Q} \times \Sigma \rightarrow \mathcal{Q}$ is the transition function. Transitions from one mode $q_{i}$ to another mode $q_{j}$ are labeled by an event $\sigma \in \Sigma$. Transitions labeled as $\sigma_{f} \in \Sigma_{\mathcal{F}}$ indicate that the transition is a faulty transition.

The HA operational modes and transitions for the patient are presented in Figure 2. Three automata modes define the normal operation for the system. These modes were selected for its differentiated dynamics and significance during operation. Transitions between and to these modes are labeled $\Sigma_{S} \cup \Sigma_{C}=\left\{\sigma_{1}, \sigma_{2}, \ldots, \sigma_{13}\right\}$ and include input patient information about meals and exercise, and events triggered by the consistency of residuals. Transitions to faulty modes are possible depending on the current operation mode. The set $\Sigma_{\mathcal{F}}=\left\{\sigma_{f 1}, \sigma_{f 2}, \ldots, \sigma_{f 7}\right\}$ represent the fault events. Some of these faults may be structural (faults in the actuator) such as not eating rescue $\mathrm{CHO}$ or injecting inappropriate boluses for meals.

\subsection{Online Diagnoser}

The online diagnoser is responsible of the mode change detection within the HA. At every sampling time $k$, the patient observable events $\Sigma_{C}$ and the generated residuals are processed and used by the hybrid diagnosis to detect specific modes or faults. Patient-inthe-loop faults are detected when a triggered transition leads to one of the faulty modes, see Figure 2. 


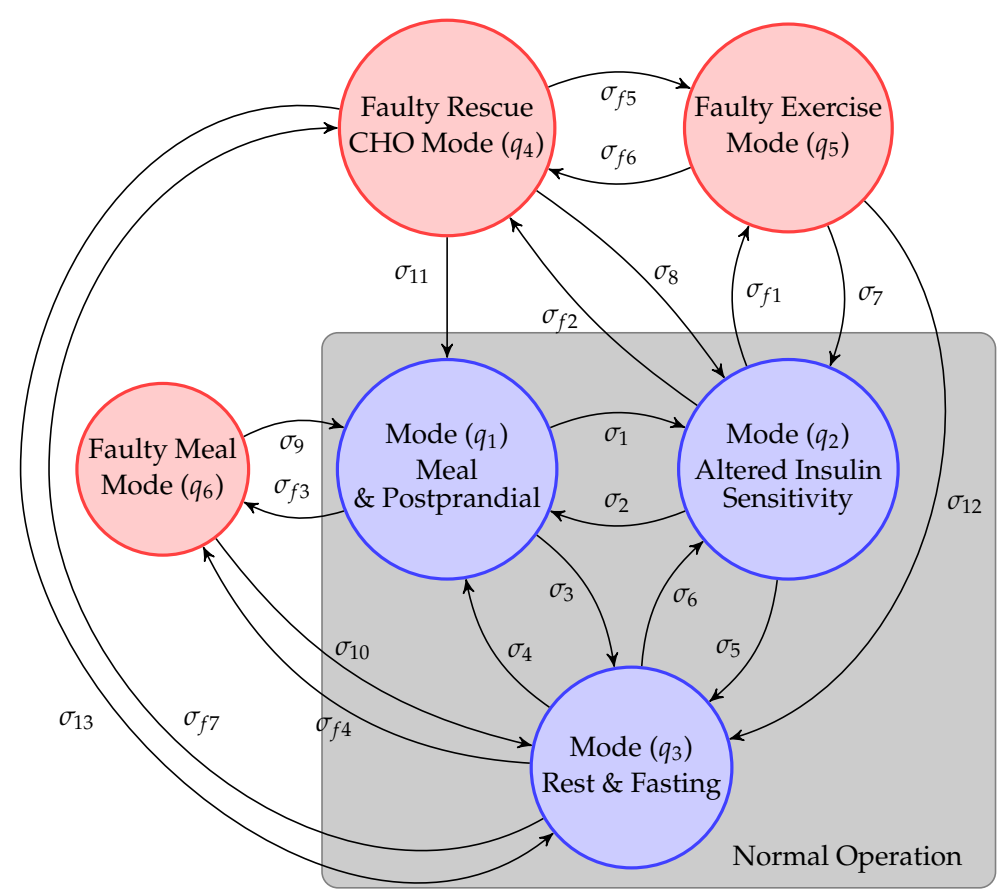

Figure 2. Component automata. The hybrid model of the automata includes three normal operation modes and considers three faulty modes.

The consistency of the residuals is checked at every sampling time against the designed thresholds. A potential mode change is detected if the residuals violate any of the thresholds. Then, a binary signature vector $(\mathbf{F})$ is generated and checked with the admissible rows of the fault signature matrix (FSM). The FSM is a binary matrix that displays the signatures required to move from one automaton mode to another. A signature is used to describe a configuration of symptoms that may lead to a transition. Each of the rows of the FSM are related to each of the possible automaton transitions $\mathcal{T}$. Hence, the FSM has as many rows as possible events $\sigma\left(n_{\sigma}=20\right)$ and as many columns as existing automaton modes $\left(n_{m}=6\right)$.

$$
\mathbf{F S M}=\left[\begin{array}{cccc}
f_{1,1} & f_{1,2} & \cdots & f_{1, n_{m}} \\
f_{2,1} & f_{2,2} & \cdots & f_{2, n_{m}} \\
\vdots & \vdots & \vdots & \vdots \\
f_{n_{\sigma}, 1} & f_{n_{\sigma}, 2} & \cdots & f_{n_{\sigma}, n_{m}}
\end{array}\right]
$$

This can be viewed as having a total of $n_{m}$ different subsets of FSMs for each of the HA modes. For example, the FSM associated to the mode $q_{1}$ is as follows

$$
\mathbf{F S M}_{\mathbf{q}_{1}}:=\left[\begin{array}{cccccc}
0 & f_{\sigma_{1}, q_{2}} & 0 & 0 & 0 & 0 \\
0 & 0 & f_{\sigma_{3}, q_{3}} & 0 & 0 & 0 \\
0 & 0 & 0 & 0 & 0 & f_{\sigma_{f 3}, q_{6}}
\end{array}\right]=\left[\begin{array}{cccccc}
0 & 1 & 0 & 0 & 0 & 0 \\
0 & 0 & 1 & 0 & 0 & 0 \\
0 & 0 & 0 & 0 & 0 & 1
\end{array}\right]
$$

The $\mathbf{F S M}_{\mathbf{q} 1}$ will only be used when the current HA mode is $q_{1}$. Then, the binary signature vector $\mathbf{F}_{1 \times n_{m}}$ is constructed at every sampling time. Elements $f_{i}$ of $\mathbf{F}$ are computed as

$$
f_{i}= \begin{cases}1, & \text { if } \prod_{i=1}^{M}\left(t_{r_{i}}^{l}<0 \vee t_{r_{i}}^{u}>0\right)=1 \\ 0, & \text { otherwise }\end{cases}
$$

where $i=1, \cdots, n_{m}$ indicates the binary residual generated by the appropriate observers for the current operation mode at time instant $k, M \leq n_{o}$ accounts for possible residual 
combinations that may be used to generate the binary signal, $n_{0}$ is the total number of observers, $t_{r_{i}}^{l}$ and $t_{r_{i}}^{u}$ are the lower and upper interval residual bands generated by the $i$-th zonotopic observer. Essentially, if the observer predicted band does not include the zero residual, then the real system behavior cannot be explained and a fault is triggered by that residual signal. The vector $\mathbf{F}$ is then compared element-wise to each of the rows of the appropriate $\mathbf{F S M}_{q_{i}}$. If the signature $\mathbf{F}$ is not matched by any of the rows of the particular FSM $_{q_{i}}$, then transition detection is not possible and the HA stays at the current mode.

\subsection{Observable Events Processing}

Using a binary transition and fault detection system might not be enough for detection if several signatures have the same binary combination, i.e., they are not isolable. For that reason, the transitions may be aided with external information, such as input events, the sign of the residuals and by the value and trend of the measured variables. In this work, the system makes use of patient information, if available, such as meal/exercise announcements. Particularly, we consider the following transitions based on the FSM and patient information:

1. Meal and Postprandial Mode Detection: Transitions between the meal/postprandial mode $\left(q_{1}\right)$ and the resting/fasting mode $\left(q_{3}\right)$ happen when in normal operation. If meals are announced, the system can instantly switch to the meal/postprandial mode and if in a designed period of $15 \mathrm{~min}$ the meal observer does not explain the system behavior, a transition to the meal faulty mode $\left(q_{6}\right)$ will be triggered. In the situation where both meal and rest residuals are consistent, the system returns to the resting/fasting operational mode.

2. Altered Sensitivity Periods Detection: Transitions $\sigma_{1}, \sigma_{2}, \sigma_{5}$, and $\sigma_{6}$ to and from the changed insulin sensitivity mode $\left(q_{2}\right)$ are considered. Similarly to $\sigma_{4}$, the transition events $\sigma_{1}$ and $\sigma_{6}$ are activated if the altered sensitivity residual is consistent with the observations. In this approach, an altered sensitivity period is caused when performing aerobic physical activity. Then, if exercise is announced, a transition to the $q_{2}$ mode can instantly happen if the altered sensitivity residuals are consistent. Transition events $\sigma_{2}$ and $\sigma_{5}$ are triggered if the meal or the rest residuals consistency are valid and the altered insulin sensitivity residual is not. If the consistency of both residuals generated by the meal and rest observers are satisfied, then the binary FSM cannot tell which transition to execute. In such a case, prior patient inputs can be used to decide if a transition needs to be made. If the residual from the altered sensitivity observer is no longer consistent, the residual with minimum absolute value is picked as the next transition event to be processed.

3. Misestimated Meals Detection: The AP used in this work requires patients to announce meals. Announcing a meal means that the patient needs to provide two inputs: (1) the time when the meal is going to be consumed and (2) the quantity of $\mathrm{CHO}$ in grams of that meal. The detection of a misestimated meal $\left(q_{6}\right)$ involves the transition events $\sigma_{9}, \sigma_{10}, \sigma_{f 3}$ and $\sigma_{f 4}$. Transition towards $q_{6}$ is possible through events $\sigma_{f 3}$ and $\sigma_{f 4}$. If a meal is not announced while being on mode $q_{3}$ and the rest observer is not consistent, then the CGM signal is checked. If CGM is higher than $160 \mathrm{mg} / \mathrm{dL}$ and the trend for the last $30 \mathrm{~min}$ is positive, a transition to mode $q_{6}$ happens. Contrarily, if the meal is announced, then transition event $\sigma_{f 3}$ might be triggered when in mode $q_{1}$, if the meal observer is not consistent.

4. Missed Rescue Carbohydrates Detection: Two types of rescue $\mathrm{CHO}$ are considered: (1) feed-forward rescue $\mathrm{CHO}$ when exercise is announced and (2) rescue CHO suggested by the feedback controller. This control action is a source of potential patient-inthe-loop faults in free living conditions. Patients may forget to consume the suggested $\mathrm{CHO}$ or they will simply not consume them for other physiological reasons, such as weight gain. Transitions to the faulty rescue $\mathrm{CHO}$ mode $\left(q_{4}\right)$ are expected through events $\sigma_{f 6}$ or $\sigma_{f 7}$, which are only checked when the controller triggers a recommendation of $\mathrm{CHO}$. Transitions $\sigma_{8}, \sigma_{11}$ and $\sigma_{13}$ return the HA to normal operational modes, 
respectively to $q_{2}, q_{1}$ and $q_{3}$. These transitions are exclusive, meaning that if any of the meal, rest or altered sensitivity observer residuals are consistent, a transition to any of the aforementioned normal operation modes will be triggered.

\section{Modeling of T1D Patients}

In this section, the model used for the design of observers is presented. Several dynamical models are available in the literature to describe normal and impaired glucose metabolism in humans. One of the early models and most likely the most used is the socalled minimal model [38], which only consists of three state variables and is able to capture some of the underlying glucose dynamics. Since then, many other models have appeared such as the more complex Food and Drug Administration (FDA) accepted UVA/Padova T1D Simulator [39]. Selecting a model for the design of observers is crucial and one has to trade off precision and complexity with simplicity and usability. Particularly, complex glucose models may present observability issues because only one variable is measured. For that reason, we use a modified version of the Hovorka nonlinear model [40], which is of intermediate complexity [41].

\subsection{The Reduced Hovorka Model}

The model used in this work is a reduced version of the nonlinear Hovorka model [40] as proposed in [42]. Figure 3 shows the whole block diagram of both model versions. It is a compartmental model with four main sub-systems: carbohydrate absorption, subcutaneous insulin absorption, insulin action on glucose uptake and removal, and the BG dynamics.

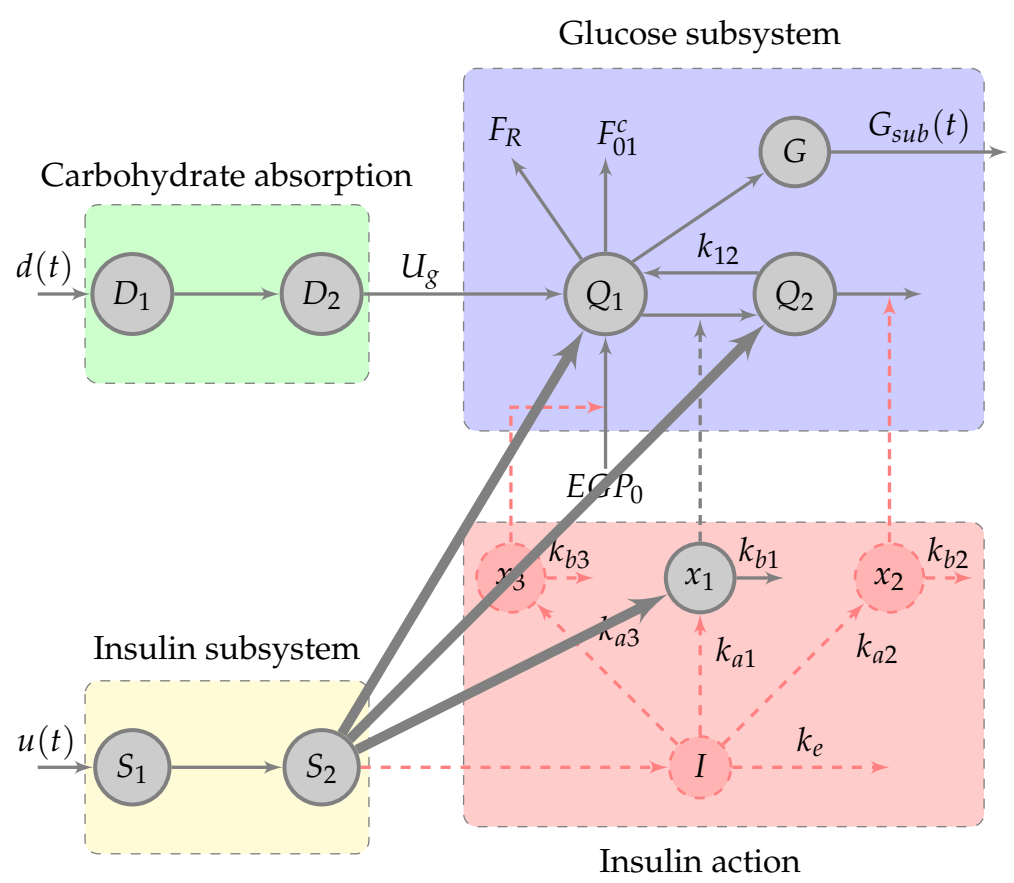

Figure 3. Block diagram of the reduced Hovorka nonlinear model. The dashed red nodes and arrows indicate the structural relations removed from the original nonlinear Hovorka model. The thicker black lines denote new relations in the reduced model.

The simplified model excludes the states $I(t), x_{2}(t)$ and $x_{3}(t)$. By doing this reduction, not only have we decreased the system complexity, but most importantly we have improved the structural behavior of the overall model. Notice the new direct connections from node $S_{2}$ to $Q_{1}$ and $Q_{2}$. Those connections greatly simplify any observability issues since 
more relations between different states are considered. The removed states define insulin dynamics and are described by

$$
\begin{aligned}
\dot{I}(t) & =\frac{S_{2}(t)}{t_{\max I} V_{i}}-k_{e} I(t) \\
\dot{x}_{2}(t) & =-k_{a 2} x_{2}(t)+S_{I D}^{f} k_{a 2} I(t) \\
\dot{x}_{3}(t) & =-k_{a 3} x_{3}(t)+S_{I E}^{f} k_{a 3} I(t)
\end{aligned}
$$

The reduced model is obtained by considering the steady state value of the removed states into the rest of the equations. One can easily obtain the following steady state relations

$$
I^{S S}=\frac{S_{2}}{t_{\max I} V_{i} k_{e}}
$$

which leads to

$$
\begin{aligned}
& x_{2}^{s S}=\frac{S_{I D}^{f}}{t_{\max I} V_{i} k_{e}} S_{2} \\
& x_{3}^{s S}=\frac{S_{I E}^{f}}{t_{\max I} V_{i} k_{e}} S_{2}
\end{aligned}
$$

Plugging these equations into the original Hovorka model and removing the aforementioned states leads to the final model

$$
\begin{aligned}
\dot{S}_{1}(t)= & u(t)-\frac{S_{1}(t)}{t_{\max I}} \\
\dot{S}_{2}(t)= & \frac{S_{1}(t)-S_{2}(t)}{t_{\max I}} \\
\dot{x}_{1}(t)= & k_{a 1}\left(\frac{k_{a} S_{I T}^{f}}{V_{I} k_{e}} S_{2}(t)-x_{1}(t)\right) \\
\dot{Q}_{1}(t)= & -x_{1}(t) Q_{1}(t)+k_{12} Q_{2}(t)-F_{01}^{c}-F_{R}-U_{g}+ \\
& +E G P_{0}\left(1-\frac{k_{a} S_{I E}^{f}}{V_{I} k_{e}} S_{2}(t)\right) \\
\dot{Q}_{2}(t)= & x_{1}(t) Q_{1}(t)-k_{12} Q_{2}(t)+\frac{k_{a} S_{I D}^{f}}{V_{I} k_{e}} S_{2}(t) Q_{2}(t)
\end{aligned}
$$

where $u(t)$ is the exogenous insulin infusion (basal and bolus), $Q_{1}$ and $Q_{2}$ are the masses of glucose in the accessible and non-accessible (not measurable) compartments, respectively, $S_{1}$ and $S_{2}$ define the insulin absorption rate dynamics and $F_{R}$ and $F_{01}^{c}$ are defined as

$$
\begin{gathered}
F_{01}^{c}(t)= \begin{cases}F_{01} & \text { if } G(t) \geq 4.5 \mathrm{mmol} \mathrm{L}^{-1} \\
F_{01} G / 4.5 & \text { otherwise }\end{cases} \\
F_{R}(t)= \begin{cases}0.003(G(t)-9) V_{g} & \text { if } G(t) \geq 9 \mathrm{mmol} \mathrm{L}^{-1} \\
0 & \text { otherwise }\end{cases}
\end{gathered}
$$

The original Hovorka model assumes that the compartment $G$ represents the measurable glucose concentration and defines it as $G(t)=Q_{1}(t) / V_{g}$. However, one can use any other CGM model available in the literature. 
The glucose absorption sub-system can be defined separately as

$$
\begin{aligned}
& \dot{D}_{1}(t)=-\frac{D_{1}(t)}{t_{\max G}}+d(t) B \\
& \dot{D}_{2}(t)=\frac{D_{1}(t)}{t_{\max G}}-\frac{D_{2}(t)}{t_{\max G}} \\
& U_{g}(t)=\frac{D_{2}(t)}{t_{\max G}}
\end{aligned}
$$

where $U_{g}$ is the rate of carbohydrate absorption, $d(t)$ is the input meal content in grams and $D_{1}$ and $D_{2}$ are glucose compartments. If meals are considered in the model, the state vector is simply extended with states $D_{1}$ and $D_{2}$.

\subsection{Model Parameters and Individualization}

For the purpose of state estimation, we use the model constants and mean parameter values presented in [40] and summarized in Table 1.

Table 1. Model parameters.

\begin{tabular}{lcl}
\hline Parameter & Value & Description \\
\hline$B(\mathrm{~kg})$ & Individualized & Weight \\
$k_{12}\left(\mathrm{~min}^{-1}\right)$ & 0.066 & Transfer rate \\
$k_{a 1}\left(\mathrm{~min}^{-1}\right)$ & 0.006 & Deactivation rate \\
$k_{a 2}\left(\mathrm{~min}^{-1}\right)$ & 0.06 & Deactivation rate \\
$k_{a 3}\left(\mathrm{~min}^{-1}\right)$ & 0.03 & Deactivation rate \\
$k_{e}\left(\mathrm{~min}^{-1}\right)$ & 0.138 & Time constant of insulin elimination \\
$V_{i}(\mathrm{~L})$ & $0.12 \mathrm{BW}$ & Insulin distribution volume \\
$V_{g}(\mathrm{~L})$ & $0.16 \mathrm{BW}$ & Glucose distribution volume \\
$S_{I T}^{f}\left(\mathrm{~L} \mathrm{~min}-1 \mathrm{mU}^{-1}\right)$ & $51.2 \times 10^{-4}$ & Insulin sensitivity on transport \\
$S_{I D}^{f}\left(\mathrm{~L} \mathrm{~min}^{-1} \mathrm{mU}^{-1}\right)$ & $8.2 \times 10^{-4}$ & Insulin sensitivity of disposal \\
$S_{I E}^{f}\left(\mathrm{~L} \mathrm{mU}^{-1}\right)$ & $520 \times 10^{-4}$ & Insulin sensitivity of $E G P_{0}$ \\
$E G P_{0}\left(\mathrm{mmol} \mathrm{kg}^{-1} \mathrm{~min}^{-1}\right)$ & 0.0161 & Endogenous glucose production at \\
& & zero insulin \\
$F_{01}\left(\mathrm{mmol} \mathrm{kg}^{-1} \mathrm{~min}^{-1}\right)$ & 0.0097 & Non-insulin-dependant glucose flux \\
$t_{\operatorname{maxI}}\left(\mathrm{min}^{-1}\right)$ & 55 & Time constant of insulin absorption \\
\hline
\end{tabular}

The model is firstly individualized by the patient's weight. The patient weight alters the insulin sensitivity parameters and the available glucose and insulin distribution volumes. However, this might not be sufficient to characterize highly varying patients and therefore model predictions will be inherently erroneous. To address this individualization issue, the insulin sensitivity parameters are adapted based on the fasting patient glucose and basal infusion rate. Then, a new tuning parameter $\eta$ is introduced and obtained by solving the steady state of the original model

$$
\mathbf{f}\left(S_{1}, S_{2}, x_{1}, x_{2}, x_{3}, I, Q_{2}, \eta\right)=\mathbf{0}
$$

were $\mathbf{f}\left(S_{1}, S_{2}, x_{1}, x_{2}, x_{3}, I, Q_{2}, \eta\right)$ represents the non-linear model equations and $\eta$ is a factor that multiplies the insulin sensitivity $S_{I T}^{f}, S_{I D}^{f}$ and $S_{I E}^{f}$ terms. 


\subsection{Linear Parameter Varying Model}

LPV systems are linear time varying systems whose state-space system matrices depend on a vector of varying parameters. These parameters can be estimated online or measured. Then, the continuous system representation is given by

$$
\begin{aligned}
\dot{\mathbf{x}}(t) & =\mathbf{A}(\Phi(t)) \mathbf{x}(t)+\mathbf{B}(\Phi(t)) \mathbf{u}(t) \\
\mathbf{y}(k) & =\mathbf{C}(\Phi(t)) \mathbf{x}(t)+\mathbf{D}(\Phi(t)) \mathbf{u}(t)
\end{aligned}
$$

where $\mathbf{A}(\Phi(t)) \in \mathbb{R}^{n_{x} \times n_{x}}, \mathbf{B}(\Phi(t)) \in \mathbb{R}^{n_{x} \times n_{u}}, \mathbf{C}(\Phi(t)) \in \mathbb{R}^{n_{y} \times n_{x}}$ and $\mathbf{D}(\Phi(t)) \in \mathbb{R}^{n_{y} \times n_{u}}$ are the system state space matrices that depend on the varying time-dependent vector $\Phi(t) \in \mathbb{R}^{l}$. The vector of varying parameters $\Phi(t)$ depends at the same time on some measurable signals $\rho \in \mathbb{R}^{n} \Phi$, referred to as scheduling variables, that can be estimated using an appropriate scheduling function

$$
\Phi(t)=p(\rho(t))
$$

where $p: \mathbb{R}^{l} \rightarrow \mathbb{R}^{n_{\Phi}}$ is a continuous mapping. If the vector of scheduling variables depend on some internal variables, such as internal states, the system is called quasi-LPV [43]. In this work, the system is strictly proper, with input and output matrices being invariant $\mathbf{B}(\Phi(t))=\mathbf{B}, \mathbf{C}(\Phi(t))=\mathbf{C}$ and $\mathbf{D}(\Phi(t))=0$.

Particularly, the reduced Hovorka nonlinear model can be transformed into the LPV representation (12) using the nonlinear embedding approach proposed in [43]. This approach is based on embedding the system nonlinearities inside the scheduling parameters of the LPV model. Then, the following state space matrices are obtained for the reduced Hovorka model in LPV form

$$
\begin{aligned}
& \mathbf{A}(\Phi(t))=\left[\begin{array}{ccccccc}
-k_{a} & 0 & 0 & 0 & 0 & 0 & 0 \\
k_{a} & -k_{a} & 0 & 0 & 0 & 0 & 0 \\
0 & a_{31} & -k_{a 1} & 0 & 0 & 0 & 0 \\
0 & a_{41} & a_{43} & a_{44} & k_{12}+a_{45} & 0 & a_{47} \\
0 & a_{52} & a_{53} & 0 & -k_{12} & 0 & 0 \\
0 & 0 & 0 & 0 & 0 & a_{66} & 0 \\
0 & 0 & 0 & 0 & 0 & a_{76} & a_{77}
\end{array}\right] \\
& \mathbf{B}(\Phi(t))=\left[\begin{array}{lllllll}
1 & 0 & 0 & 0 & 0 & 0 & 0
\end{array}\right]^{T}
\end{aligned}
$$

with the following output equation $y(t)=Q_{2}(t) / V_{g}$ measurable from the CGM sensor. The following terms have constant values

$$
\begin{aligned}
& a_{31}=\frac{\eta k_{a} S_{I T}^{f} k_{a 1}}{V_{i} k_{e}} \\
& a_{41}=\frac{-\eta k_{a} S_{I E}^{f} E G P_{0}}{V_{i} k_{e}} \\
& a_{47}=-a_{66}=a_{76}=-a_{77}=\frac{1}{t_{\max G}}
\end{aligned}
$$


and the nonlinearities can be embedded in the following terms

$$
\begin{aligned}
& a_{43}=-Q_{1}(t) \\
& a_{44}=\frac{F_{01}^{c}(G(t))}{Q_{1}(t)}-F_{R}(G(t)) \\
& a_{45}=\frac{E G P_{0}}{Q_{2}} \\
& a_{52}=\frac{\eta k_{a} S_{I D}^{f}}{V_{I} k_{e}} Q_{2}(t) \\
& a_{53}=Q_{2}(t)
\end{aligned}
$$

Furthermore, if measurements of $\Phi$ are available, and its admissible range of operation is known

$$
\underline{\Phi}_{i} \leq \Phi_{i} \leq \bar{\Phi}_{i}, i=1, \ldots, l
$$

where $\underline{\Phi}_{i}$ and $\bar{\Phi}_{i}$ are the lower and upper bounds of each element in $\Phi$, then we can put the parameter vector $\Phi$ into polytopic form $[44,45]$

$$
\Phi \in \operatorname{Co}\left\{\omega_{1}, \omega_{2}, \ldots, \omega_{N}\right\}:=\left\{\sum_{i=1}^{N} \mu_{i} \omega_{i}: \mu_{i} \geq 0, \sum_{i=1}^{N} \mu_{i}=1\right\}
$$

where $N=\left\{1, \ldots, 2^{n_{\Phi}}\right\}$. This transformation is known as the bounding box method, because $\Phi$ is the convex hull generated by vertex $\omega_{i}$. Hence, a polytopic representation of the system is obtained by the state space matrices defined at the different vertex of the convex hull

$$
[\mathbf{A}(\Phi)] \in \operatorname{Co}\left\{\left[\mathbf{A}_{i}\right]:=\left[\mathbf{A}_{i}\left(\omega_{i}\right)\right]\right\}
$$

Using this approach, the system (12) can be represented as a weighting function of the system matrices at the polytope vertices

$$
\begin{aligned}
\dot{\mathbf{x}}(t) & =\sum_{i=1}^{2^{n} \Phi}\left(\mu_{i}(\Phi)\right)\left(\mathbf{A}_{i}(\Phi) \mathbf{x}(t)+\mathbf{B}_{i}(\Phi) \mathbf{u}(t)\right) \\
\mathbf{y}(t) & =\sum_{i=1}^{2^{n} \Phi}\left(\mu_{i}(\Phi)\right)\left(\mathbf{C}_{i}(\Phi) \mathbf{x}(t)+\mathbf{D}_{i}(\Phi) \mathbf{u}(t)\right)
\end{aligned}
$$

where $\mu(i)$ are the membership functions. Basically, at any given time instant the state space system description is obtained by a linear interpolation of the system matrices at the polytope vertices. The weighting function is defined as in [46]

$$
\mu_{i}(\Phi)=\prod_{j=1}^{2^{n} \Phi} \xi_{i j}\left(\eta_{0}^{j}, \eta_{1}^{j}\right), \forall i=\left\{1, \ldots, 2^{n_{\Phi}}\right\}
$$

with

$$
\begin{aligned}
& \xi_{i j}\left(\eta_{0}^{j}, \eta_{1}^{j}\right)= \begin{cases}\eta_{0^{j}}^{j} & \text { if } \bmod \left(n, 2^{m}\right) \in\left\{1, \ldots, 2^{j-1}\right\} \\
\eta_{1}^{j}, & \text { otherwise }\end{cases} \\
& \eta_{0}^{j}=\frac{\bar{\Phi}_{j}-\Phi(k)}{\bar{\Phi}_{j}-\underline{\Phi}_{j}} \\
& \eta_{1}^{j}=1-\eta_{0}^{j}
\end{aligned}
$$


where $j=1, \ldots, n_{\Phi}$ and each element of the vector $\Phi$ is known and varies in a known interval $\Phi_{j}(k) \in\left[\underline{\Phi}_{j}, \bar{\Phi}_{j}\right]$. The scheduling variables for the reduced Hovorka model can be defined as

$$
\Phi(t)=\left[Q_{1}(t) \quad Q_{2}(t)\right]
$$

leading to four linear time invariant (LTI) systems that will conform the vertices of the polytope. Notice that this representation is not unique and other scheduling variables can be chosen depending on the model. The operational range for these variables is attached in Table 2.

Table 2. Limits for the system scheduling variables.

\begin{tabular}{ccc}
\hline Variable & Minimum & Maximum \\
\hline$Q_{1}(\mathrm{mmol})$ & 10 & 400 \\
$Q_{2}(\mathrm{mmol})$ & 10 & 400 \\
\hline
\end{tabular}

The original continuous time system can be discretized using a number of discretization methods. In this work, we use the zero order hold approach with a sampling time of $5 \mathrm{~min}$, which is a common measurement rate provided by CGM devices. Then, the continuous polytopic system (20) takes the following discrete-time representation

$$
\begin{aligned}
\mathbf{x}(k+1) & =\sum_{i=1}^{2^{n} \Phi}\left(\mu_{i}(\Phi)\right)\left(\mathbf{A}_{d, i}(\Phi) \mathbf{x}(k)+\mathbf{B}_{d, i}(\Phi) \mathbf{u}(k)\right) \\
\mathbf{y}(k) & =\sum_{i=1}^{2^{n} \Phi}\left(\mu_{i}(\Phi)\right)\left(\mathbf{C}_{d, i}(\Phi) \mathbf{x}(k)+\mathbf{D}_{d, i}(\Phi) \mathbf{u}(k)\right)
\end{aligned}
$$

where $\mathbf{A}_{d, i}(\Phi) \in \mathbb{R}^{n_{x} \times n_{x}}, \mathbf{B}_{d, i}(\Phi) \in \mathbb{R}^{n_{x} \times n_{u}}, \mathbf{C}_{d, i}(\Phi) \in \mathbb{R}^{n_{y} \times n_{x}}$ and $\mathbf{D}_{d, i}(\Phi) \in \mathbb{R}^{n_{y} \times n_{u}}$ are the discretized state space matrices for the $i$-th vertex.

\section{Residual Generation}

The AP systems are characterized by having few sensors and actuators. Therefore, to monitor the system and patient state, observers are designed for the purpose of generating residuals. However, only little information is available for AP systems. In most cases, the only information available from sensors is the CGM glucose measurements. To tackle this limitation, the design of a robust state estimator for residual generation has been considered in this section.

\subsection{Design Method}

Residual generation is based on the state estimation using the polytopic LPV model (26) by means of a Kalman filter of the following form

$$
\begin{aligned}
\hat{\mathbf{x}}(k+1)= & \sum_{i=1}^{2^{n} \Phi}\left(\mu_{i}(\Phi)\right)\left(\mathbf{A}_{d, i}(\Phi) \mathbf{x}(k)+\mathbf{B}_{d, i}(\Phi) \mathbf{u}(k)\right)+ \\
& +\mathbf{L}(\Phi)(y(k)-\hat{y}(k))
\end{aligned}
$$

were $\hat{\mathbf{x}} \in \mathbb{R}^{n_{x}}$ are the estimated states and $\mathbf{L}(\Phi) \in \mathbb{R}^{n_{x} \times n_{y}}$ is the observer gain defined as

$$
\mathbf{L}(\Phi)=\sum_{i=1}^{2^{n} \Phi} \mu_{i}(\Phi) \mathbf{L}_{i}
$$


and $\mathbf{L}_{i}$ are the observer gains for each one of the systems at the vertices of the polytope. Prior to the observer design, the observability property is checked. A total of $2^{n} \Phi$ observability matrices are generated, for each one of the vertex polytopic representations

$$
\mathbf{O}=\left[\begin{array}{c}
\mathbf{C}_{d, i}(\Phi) \\
\mathbf{C}_{d, i}(\Phi) \mathbf{A}_{d, i}(\Phi) \\
\vdots \\
\mathbf{C}_{d, i}(\Phi) \mathbf{A}_{d, i}(\Phi)^{n_{x}-1}
\end{array}\right]
$$

then, the system is observable if each observability matrix is of full rank, i.e., $\operatorname{rank}(\mathbf{O})=n_{x}$.

The observer gain can be designed by solving the associated LQC dual problem [47]. Given the system description (26), tuning matrices $\mathbf{Q}=\mathbf{Q}^{T}=\mathbf{H}^{T} \mathbf{H} \geq 0, \mathbf{R}=\mathbf{R}^{T}>0$ and the performance bound $\gamma$. Then, the observer gains for the polytopic system are obtained by finding $\mathbf{Y}$ and $\mathbf{W}_{i}$ through the solution of the following LMI minimization problem

$$
\begin{gathered}
\min _{\gamma, \mathbf{Y}=\mathbf{Y}^{T}, \mathbf{W}} \gamma \\
\text { subject to } \\
{\left[\begin{array}{cc}
\gamma \mathbf{I}_{n} & \mathbf{I}_{n} \\
\mathbf{I}_{n} & \mathbf{Y}
\end{array}\right]>0} \\
{\left[\begin{array}{cccc}
-\mathbf{Y} & \mathbf{Y} \mathbf{A}_{d, i}-\mathbf{W}^{T} \mathbf{C}_{d, i} & \mathbf{Y} \mathbf{H}^{T} & \mathbf{W}^{T} \\
\mathbf{A}_{d, i}^{T} \mathbf{Y}-\mathbf{C}_{d, i}^{T} \mathbf{W} & -\mathbf{Y} & 0 & 0 \\
\mathbf{H Y} & 0 & \mathbf{I}_{n_{x}} & 0 \\
\mathbf{W} & 0 & 0 & -\mathbf{R}^{-1}
\end{array}\right]<0} \\
{\left[\begin{array}{ccc}
-r \mathbf{Y} & q \mathbf{Y}+\mathbf{A}_{d, i}^{T} \mathbf{Y}-\mathbf{C}_{d, i}^{T} \mathbf{W} \\
q \mathbf{Y}+\mathbf{Y A}_{d, i}-\mathbf{W C}_{d, i} & -r \mathbf{Y}
\end{array}\right]<0}
\end{gathered}
$$

where $q=0$ and $r=1$ are the center and radius of a unitary circle respectively and $\mathbf{I}_{n_{x}}$ is the identity matrix of size $n_{x}$. Note that constraints (33) are not mandatory to solve the original LQC problem [47]. However, they guarantee the stability of the observer system.

In this paper, the process disturbances $\left(\omega \in \mathbb{R}^{n_{x}}\right)$ and measurement noise $\left(v \in \mathbb{R}^{n_{y}}\right)$ are unknown but assumed to be bounded and represented by zonotopes

$$
\begin{array}{r}
\mathcal{W}=\left\{\omega_{k} \in \mathbb{R}^{n_{x}}:\left|\omega_{k}-c_{\omega}\right| \leq \bar{\omega}, c_{\omega} \in \mathbb{R}^{n_{x}}, \bar{\omega} \in \mathbb{R}^{n_{x}}\right\}=\left\langle c_{\omega}, R_{\omega}\right\rangle \\
\mathcal{V}=\left\{v_{k} \in \mathbb{R}^{n_{y}}:\left|v_{k}-c_{v}\right| \leq \bar{v}, c_{v} \in \mathbb{R}^{n_{y}}, \bar{v} \in \mathbb{R}^{n_{y}}\right\}=\left\langle c_{v}, R_{v}\right\rangle
\end{array}
$$

where $c_{\omega}$ and $c_{v}$ are the centers of the process disturbances and measurement noise zonotopes, with the generator matrices being $R_{\omega} \in \mathbb{R}^{n_{x} \times n_{x}}$ and $R_{v} \in \mathbb{R}^{n_{y} \times n_{y}}$. Then, according to [48] the polytopic observer in (27) can be converted into a zonotopic state estimation observer by computing the center and the generator matrix of the state observer, i.e., $\hat{\mathcal{X}}=\left\langle c_{x}, R_{x}\right\rangle$, at every single step as follows

$$
\begin{aligned}
& c_{x}(k+1)=c_{p}(k)+\mathbf{L}\left(y(k)-\sum_{i=1}^{2^{n} \Phi}\left(\mu_{i}(\Phi) \mathbf{C}_{\mathbf{i}}\right) c_{p}(k)\right) \\
& R_{x}(k+1)=\left[\left(\mathbf{I}-\mathbf{L} \sum_{i=1}^{2^{n}}\left(\mu_{i}(\Phi) \mathbf{C}_{\mathbf{i}}\right)\right) R_{p}(k)-\mathbf{L} E_{v}\right]
\end{aligned}
$$


with $\mathbf{L}$ being defined in Equation (28) and

$$
\begin{aligned}
c_{p}(k+1) & =\sum_{i=1}^{2^{n}}\left(\mu_{i}(\Phi)\right)\left(\mathbf{A}_{i}(\Phi) \mathbf{x}(k)+\mathbf{B}_{i} \mathbf{u}(k)\right) \\
R_{p}(k+1) & =\left[\sum_{i=1}^{2^{n} \Phi}\left(\mu_{i}(\Phi)\right)\left(\mathbf{A}_{i}(\Phi)\right) R_{x}(k) \quad E_{\omega}\right]
\end{aligned}
$$

where $E_{\omega}$ and $E_{v}$ are the distribution matrices of the state disturbance and measurement noise vectors, respectively.

\subsection{The Bank of Observers}

The bank of interval zonotopic observers is designed to generate appropriate residual signals. The residual signals have to be sensitive to specific mode changes. Therefore, several observers based on variations of the reduced Hovorka model have been considered. Each of the observers tackle one specific mode of operation. A total of four different observers have been designed $\left(n_{0}=4\right)$ :

1. Meal observer: Observer designed to track meal and postprandial periods for announced meals. It allows the system to detect faults in meal estimations and erroneous boluses. It includes the whole Hovorka model with two extended states related to

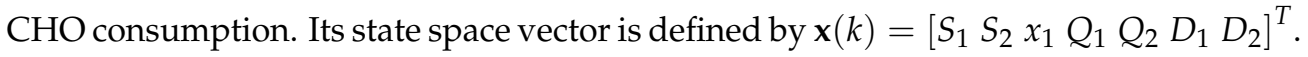
The CHO model time constant $t_{\max }$ is set to $40 \mathrm{~min}$ as in [40];

2. Resting and fasting observer: Observer designed to track night and in-between distur-

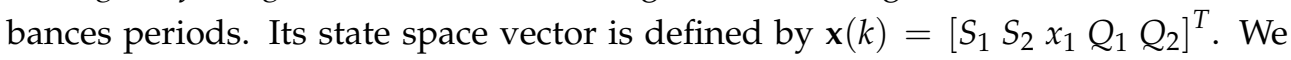
assume that sleeping periods are characterized by having no disturbances affecting the system and/or the effect of previous disturbances is small. For this reason, the observer is designed considering only insulin inputs. Consistent estimations should be provided by this observer during periods similar to steady state periods, i.e., resting or fasting periods;

3. Altered insulin sensitivity observer: Observer designed to track periods with increased sensitivity, for example during aerobic exercise sessions. The model parameter $\eta$ has been increased by a trial and error procedure to match simulated aerobic exercise sessions based on clinical results [32]. Its state space vector is defined by $\mathbf{x}(k)=$

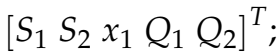

4. Rescue carbohydrates observer: Observer designed to detect a specific patient-in-the-loop control action. The presented control approach uses $\mathrm{CHO}$ as a counter-regulatory action to prevent hypoglycemia. This observer takes the insulin and $\mathrm{CHO}$ controller actions as its inputs. The goal of the observer is to monitor the adherence of the patient

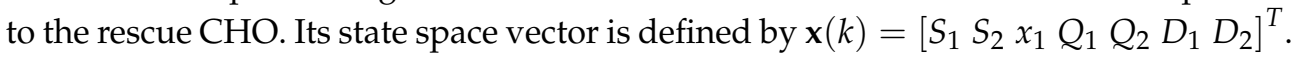
The CHO model time constant $t_{\max }$ is set to $20 \mathrm{~min}$ as in [27].

Each of the designed observers return a full state space interval estimation $\hat{\mathbf{x}}_{1}, \hat{\mathbf{x}}_{2}, \ldots \hat{\mathbf{x}}_{n_{0}}$. Then, using those estimations, the interval residuals are computed based on the state bounding zonotope $\hat{\mathcal{X}}(k)$ with the following residual center $\left(c_{r}\right)$ and generator matrix $R_{r}$

$$
\begin{gathered}
c_{r}(k)=y(k)-\sum_{i=1}^{2^{n} \Phi}\left(\mu_{i}(\Phi) \mathbf{C}_{i}\right) \hat{\mathbf{x}}(k) \\
R_{r}(k)=\left[-\sum_{i=1}^{2^{n} \Phi} \mathbf{C}_{i} R_{x}(k)-E_{v}\right]
\end{gathered}
$$

Hence, the FD test is based on checking the following condition

$$
0 \notin<c_{r}(k) R_{r}(k)>
$$


A fault (or change of mode) is triggered when 0 is not included in the zonotope $<c_{r}(k) R_{r}(k)>$. To reduce the computational burden, condition (42) can be simplified to check whether or not 0 is included in a box enclosing the zonotope

$$
0 \notin<c_{r}(k) b\left(R_{r}(k)\right)>
$$

where $b\left(R_{r}(k)\right)=\operatorname{diag}\left(\left\|R_{r}(k)\right\|_{1}\right)$ and $\|\cdot\|_{1}$ is the element by element absolute value operator. Then, the zonotopic interval upper and lower bands used for FD in the FSM in Equation (3) are defined as

$$
\begin{aligned}
& t_{r_{i}}^{u}(k)=c_{r}(k)+\left\|R_{r}(k)\right\|_{1} \\
& t_{r_{i}}^{l}(k)=c_{r}(k)-\left\|R_{r}(k)\right\|_{1}
\end{aligned}
$$

The overall method scheme of the fault diagnosis system is shown in Figure 4. The observer design problem is solved offline and the observer gains are obtained. For the online observer iterations, a continuous-discrete approach can be used. With this approach the a priori state estimation is obtained by integrating the original non-linear model, for example using the ode 45 function from Matlab. Then, the polytopic observer gain is found by interpolation using (28). Residuals are then generated for each of the observers and fed to the signature analysis module.

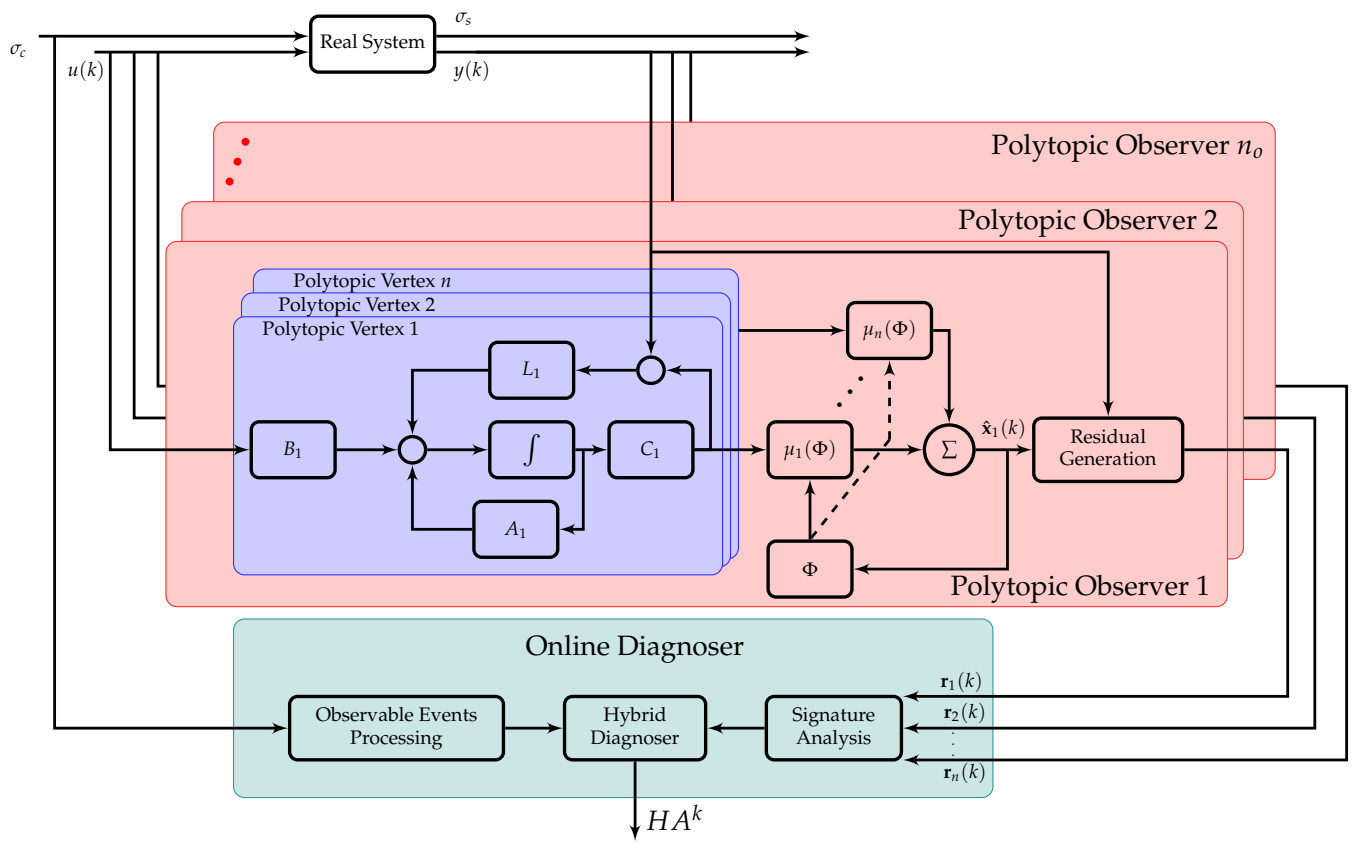

Figure 4. Conceptual diagram of the patient monitoring and FD system.

\section{Results}

\subsection{In Silico Benchmarks}

To test the robustness and precision of the monitoring system, two in silico benchmarks have been developed. Each of the benchmarks were designed to check the proper behavior of the system and its ability to detect different patient-in-the-loop modes and faults. All simulations have been executed with Matlab R2019a using an AMD Ryzen $3800 \times 3.9 \mathrm{GHz}$ processor with $32 \mathrm{~GB}$ of RAM.

The FDA accepted UVa/Padova T1D Simulator (v3.2) [49] was used to evaluate the strategy. All of the benchmarks share some scenario settings, which include a virtual cohort of 10 adult patients. Scenarios last 4 days and 3 mixed meals of 60,80 , and 70 grams are included each day at 8:00,13:00, and 21:00, respectively. Intra-subject variability in insulin absorption was included by assuming a $\pm 30 \%$ in parameters $\left(k_{d}, k_{a 1}, k_{a 2}\right)$, and variability 
in insulin sensitivity was modeled by a sinusoidal pattern in parameters $\left(V_{m x}, k_{p 3}\right)$ [50]. The first scenario is designed to evaluate performance when only meal disturbances appear. The second scenario includes one session of aerobic exercise of heavy intensity $(60 \%$ $\mathrm{VO}_{2 \max }$ ) during the second scenario day at 18:00 for a total duration of $50 \mathrm{~min}$. Exercise was included into the simulation by using a previous developed model, which was fit to clinical data, that increases insulin sensitivity [32,51].

\subsection{Patient Mode Detection}

Scenarios 1 and 2 were used to perform four complete simulations, which consider different patient-in-the-loop faults. The simulations have the following characteristics: (1) scenario 1 without faults, (2) scenario 2 without faults, (3) scenario 1 with patient misestimated meals, and (4) scenario 2 with misestimated meals and missed rescue $\mathrm{CHO}$. In total, 480 meals were consumed and 20 exercise sessions were performed across all simulations. Faults were introduced in a total of 160 meals, with over- and underestimations of $\pm 60 \%$, and feed-forward rescue $\mathrm{CHO}$ were also simulated with patient-in-the-loop faults in 10 occasions.

Meals were correctly detected in 461 occasions out of the total 480 meals across all simulations, resulting in a high sensitivity of $96.0 \%$. Transitions towards the meal mode $q_{1}$ were triggered by the announcements and validated by the meal residual observer, resulting in a detection time from the start of the event of $13 \mathrm{~min}$, while the average time in meal and postprandial period was $158 \mathrm{~min}$. All exercise sessions were correctly detected without false positives/negatives. The effect of exercise is known to last during the following hours after an exercise session, this is also reflected in the time the system remained on mode $q_{2}$ of $328 \mathrm{~min}$. Rest periods were considered as the periods in-between meals, without periods where exercise happened, or during night. No detection time for these periods is provided since there is no possible definition of when these events started, average time in this period was $328 \mathrm{~min}$.

Transitions to faulty modes only happened in simulations 3 and 4 . Faults in the consumption of rescue $\mathrm{CHO}$, leading to transitions towards mode $q_{4}$, were recorded from simulation 4. A total of 10 missed rescue $\mathrm{CHO}$ consumption events were considered at the instant of exercise announcement at 17:40 of simulation day 2. The HA correctly detected 8 of these faulty events and was also able to transition afterwards to the $q_{2}$ mode. Two of the faulty events were not detected and the HA automaton directly transitioned to the altered insulin sensitivity mode $q_{2}$, indicating a exercise. Misestimated meals were correctly detected in 73 occasions with a sensitivity of $45.6 \%$. No transitions to state $q_{5}$ happened.

Table 3 shows the detection performance of the proposed approach for the aggregated four simulations. Figures 5-9 showcase a simulation portion (from 39 to 55 simulation hours) for the adult patient 10 . Exercise starts at time instant $42 \mathrm{~h}$ and feed-forward $\mathrm{CHO}$ are suggested at $39 \mathrm{~h}$ and $40 \mathrm{~min}$. In this, case the patient does not consume the recommended amount of $\mathrm{CHO}$ and the system transitions to a faulty $\mathrm{CHO}$ state. Notice, that the system does not know if the patient has consumed or not the suggested rescue $\mathrm{CHO}$. Afterwards, the system transitions to the exercise state and remains in that state for the following hours. This is an expected behavior of the system due to the modified insulin sensitivity that last several hours after the end of exercise. Then, at time instant $45 \mathrm{~h}$ the patient consumes a meal but the HA state remains at exercise. That is a normal behavior due to the intertwined disturbance effects on the residuals.

\subsection{Example of Controller Reconfiguration}

This section provides insights regarding the benefits of using the proposed HA model for the detection of patient-in-the-loop faults and controller reconfiguration. Particularly, we focus on the case of announced exercise. When people using an AP announce exercise, the feed-forward control can be applied in advance to mitigate the risk of exercise induced hypoglycemia. The AP used in this work has an embedded feed-forward controller for exercise [32]. This block may degrade performance if triggered by an exercise announcement 
from the patient, but no physical activity is performed afterwards. To test the benefits of controller reconfiguration, we use scenario 1 , which does not include exercise, and assume exercise at 16:00 during the second simulation day.

Table 3. Population performance metrics of patients mode transition by the HA.

\begin{tabular}{cccccll}
\hline Mode & TP & FP & FN & Sensitivity (\%) & Mean Transition Time (min) & Mean Activated Time (min) \\
\hline$q_{1}$ & 461 & 1 & 19 & 96.0 & 13 & 158 \\
$q_{2}$ & 20 & 0 & 0 & 100.0 & 55 & 288 \\
$q_{3}$ & 476 & 0 & 33 & 93.5 & - & 328 \\
$q_{4}$ & 8 & 0 & 2 & 80.0 & 17 & 83 \\
$q_{6}$ & 73 & 20 & 87 & 45.6 & 28 & 22 \\
\hline
\end{tabular}

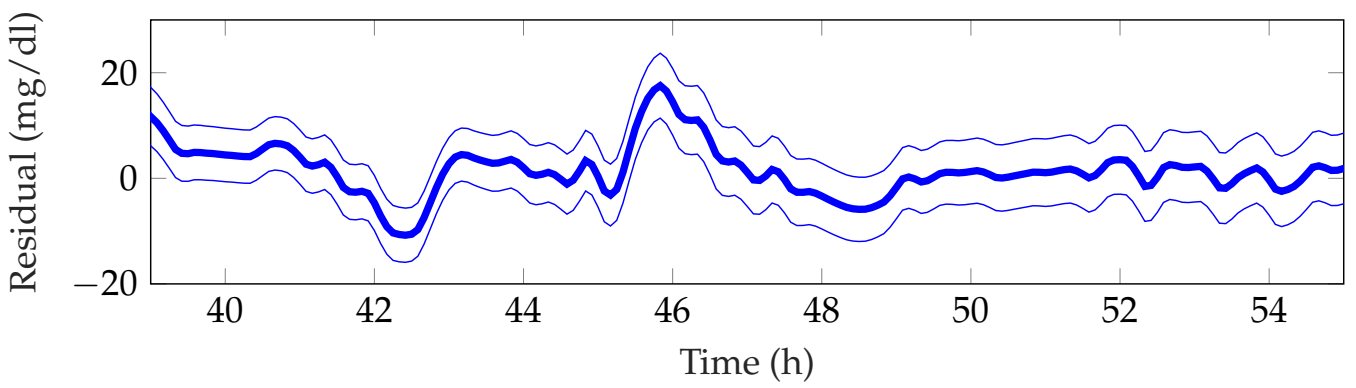

Figure 5. Representative fast and resting observer residual for the period $39-55 \mathrm{~h}$ from patient 10.

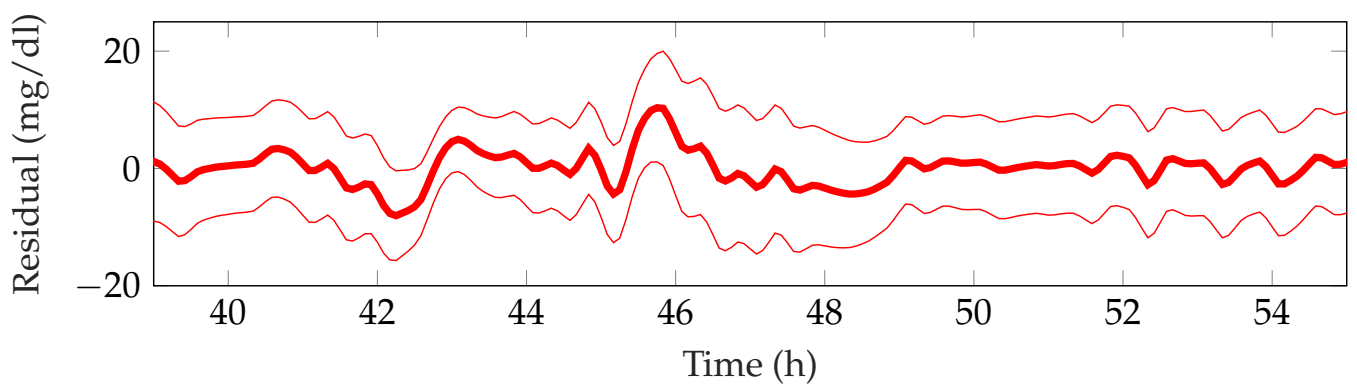

Figure 6. Representative meal observer residual for the period 39-55 h from patient 10.

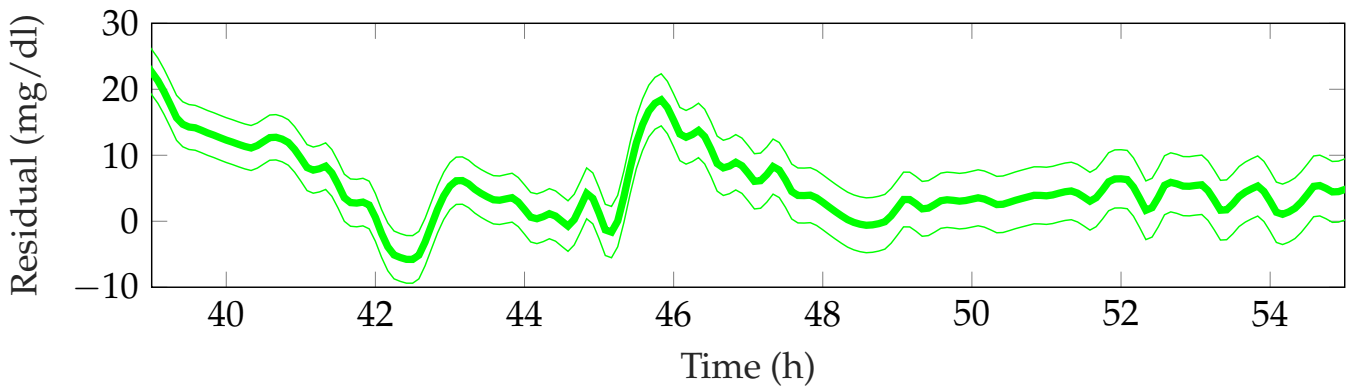

Figure 7. Representative altered insulin sensitivity observer residual for the period $39-55 \mathrm{~h}$ from patient 10.

The actions triggered by the feed-forward block are: (1) suggestion of feed-forward $\mathrm{CHO},(2)$ basal insulin reduction, (3) reduction of the next insulin bolus, and (4) decrease of the insulin controller aggressivity (lower controller gains for the next $6 \mathrm{~h}$ after the ending of the exercise session). Controller reconfiguration happens if there is a mode detection change to mode $q_{5}$ in a timely manner and the CGM is higher than $150 \mathrm{mg} / \mathrm{dL}$. We assume exercise is announced $20 \mathrm{~min}$ prior to the start [32] and we let the system monitor the patient until 
20 min after the supposed start of exercise. If the $H A^{k}$ is in a normal operational mode and there is no mode change detection to $q_{2}$ or a mode transition to $q_{5}$ happens in that period of time, then the controller resumes its original tuning and assumes that the patient is not exercising even though there was an announcement.

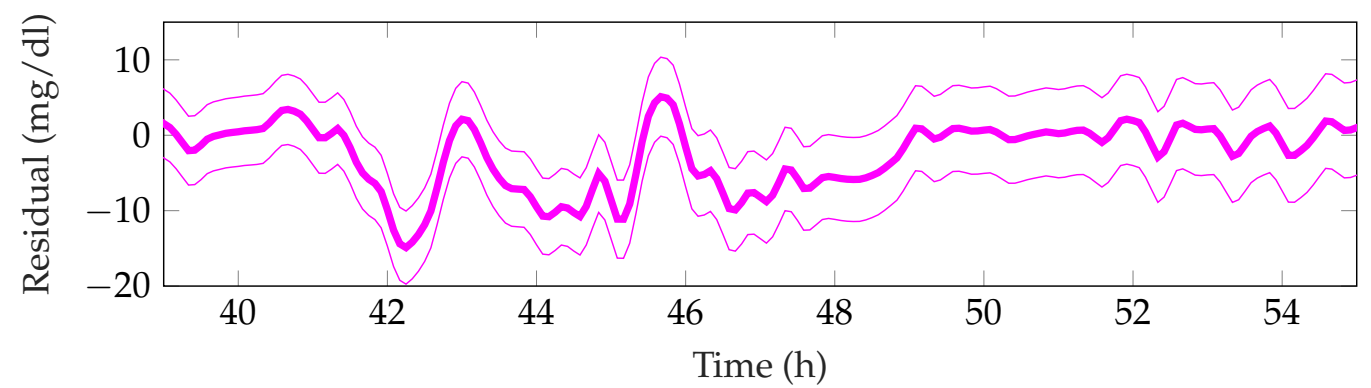

Figure 8. Representative rescue carbohydrates observer residual for the period 39-55 h from patient 10 .

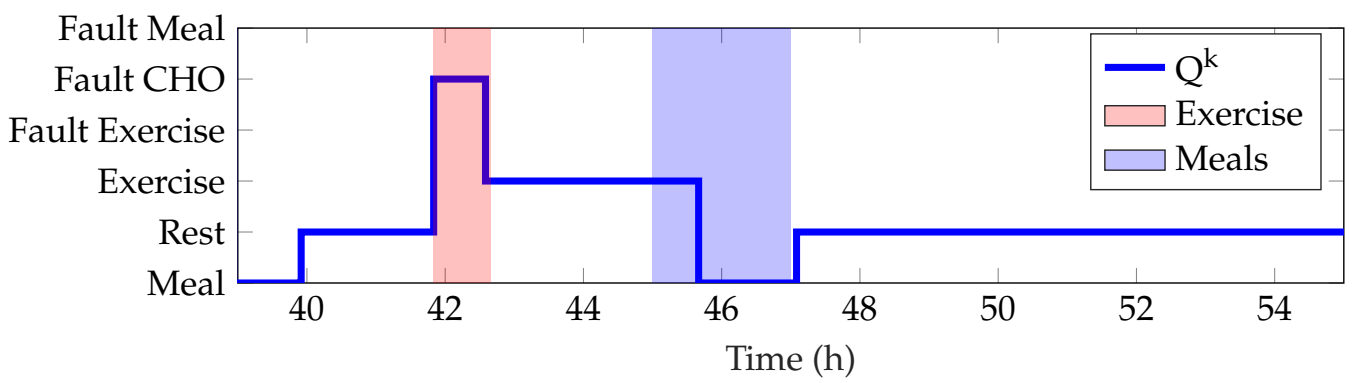

Figure 9. Representative detection of faulty rescue $\mathrm{CHO}$ by the automaton state $Q^{k}$ for patient 10 . Data shown correspond to the simulation period from 39 to $55 \mathrm{~h}$. The meal period comprises the $2 \mathrm{~h}$ following the meal and the exercise period includes the active exercise and the time in advance of the exercise announcement.

Table 4 shows the glycemic results when comparing the strategies with $(\mathrm{AP}+\mathrm{HA}$ column) and without (AP column) controller reconfiguration when faults in exercise announcement happen. Figures 10 and 11 show the CGM trajectories and insulin infusions for each case. The patient announces exercise at time instant $39 \mathrm{~h}$ and $40 \mathrm{~min}$ (day 2, 15:40). The patient is supposed to start exercising at $40 \mathrm{~h}$ (day 2, 16:00) and have dinner at $45 \mathrm{~h}$ (day 2, 21:00). Results show that reconfiguration allows for early insulin infusion to start the following meal with lower BG and allows for better and tighter BG control during the postprandial period following an exercise session.

Controller reconfiguration allowed for a tighter and better BG control during and after the false exercise session and also for the next postprandial period. Overall, time in range $70-180 \mathrm{mg} / \mathrm{dL}$ significantly increased from $38.3(34.3,47.5) \%$ to $62.0(58.4,71.5) \%$ and the mean CGM was also reduced from $211.7(172.4,231.9) \mathrm{mg} / \mathrm{dL}$ to 154.9 (121.0, 206.3) $\mathrm{mg} / \mathrm{dL}$. The risk of hyperglycemia was also minimized as reflected by the time ranges above $180 \mathrm{mg} / \mathrm{dL}$, and no risk of hypoglycemia was observed. Insulin infusion was resumed earlier upon mode detection as shown in Figure 11, allowing lower BG at the start of the second day dinner. Postprandial control was specially improved in the reconfigured controller, mainly due to the restored insulin bolus previously mitigated by the exercise feedforward actions. 
Table 4. Glycemic performance when subjects introduce faults in the exercise announcement.

\begin{tabular}{lcc}
\hline Performance Indicator & AP & AP+HA \\
\hline Mean CGM $(\mathrm{mg} / \mathrm{dL})$ & $211.7(172.4,231.9)$ & $154.9(121.0,206.3)^{*}$ \\
Median CGM $(\mathrm{mg} / \mathrm{dL})$ & $199.8(164.4,221.1)$ & $153.7(117.5,200.6)^{*}$ \\
Maximum CGM $(\mathrm{mg} / \mathrm{dL})$ & $361.3(258.1,410.3)$ & $200.9(174.0,265.3)^{*}$ \\
Minimum CGM $(\mathrm{mg} / \mathrm{dL})$ & $142.9(125.9,162.1)$ & $108.2(87.3,157.9)^{*}$ \\
\% of time CGM & & \\
$>250 \mathrm{mg} / \mathrm{dL}$ & $8.0(0.0,18.3)$ & $0.0(0.0,16.8)$ \\
$>180 \mathrm{mg} / \mathrm{dL}$ & $51.1(35.8,52.6)$ & $30.7(20.4,37.2)$ \\
$70-180 \mathrm{mg} / \mathrm{dL}$ & $38.3(34.3,47.5)$ & $62.0(58.4,71.5)^{*}$ \\
$<70 \mathrm{mg} / \mathrm{dL}$ & $0.0(0.0,0.0)$ & $0.0(0.0,0.0)$ \\
$<54 \mathrm{mg} / \mathrm{dL}$ & $0.0(0.0,0.0)$ & $0.0(0.0,0.0)$ \\
\hline
\end{tabular}

The results are median (interquartile range) during the time period between the 40th and 51th simulation hours. $* p$ value $<0.01$ (Wilcoxon signed rank test). AP refers to the system without controller reconfiguration and $\mathrm{AP}+\mathrm{HA}$ refers to the system with controller reconfiguration.

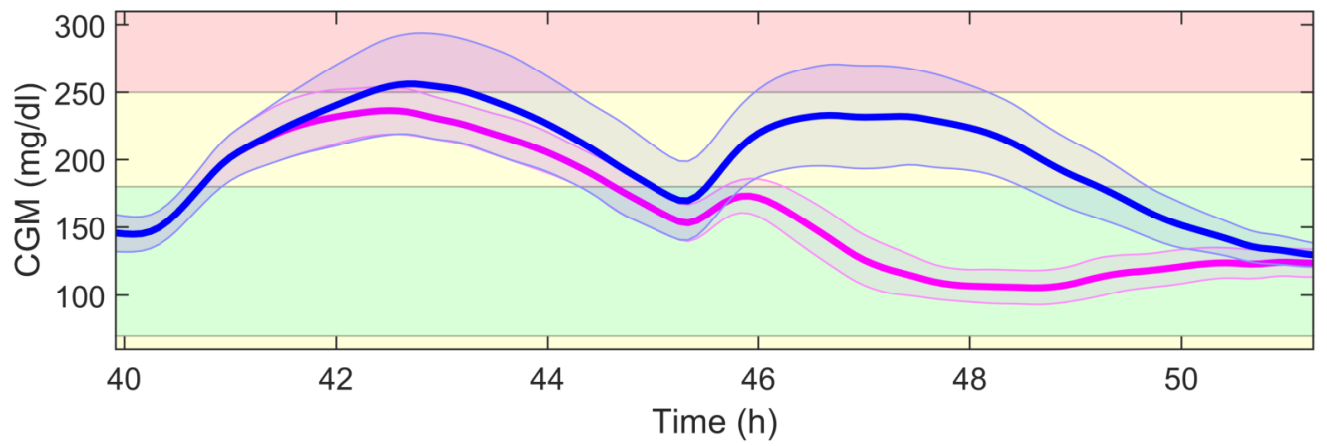

Figure 10. Population CGM trajectory (MEAN \pm STD) when an exercise announcement patient-inthe-loop fault affects the system. The blue curve represents the controller without reconfiguration and the magenta curve represents the controller with reconfiguration. The exercise announcement occurs at time instant $39 \mathrm{~h}$ and $40 \mathrm{~min}$.

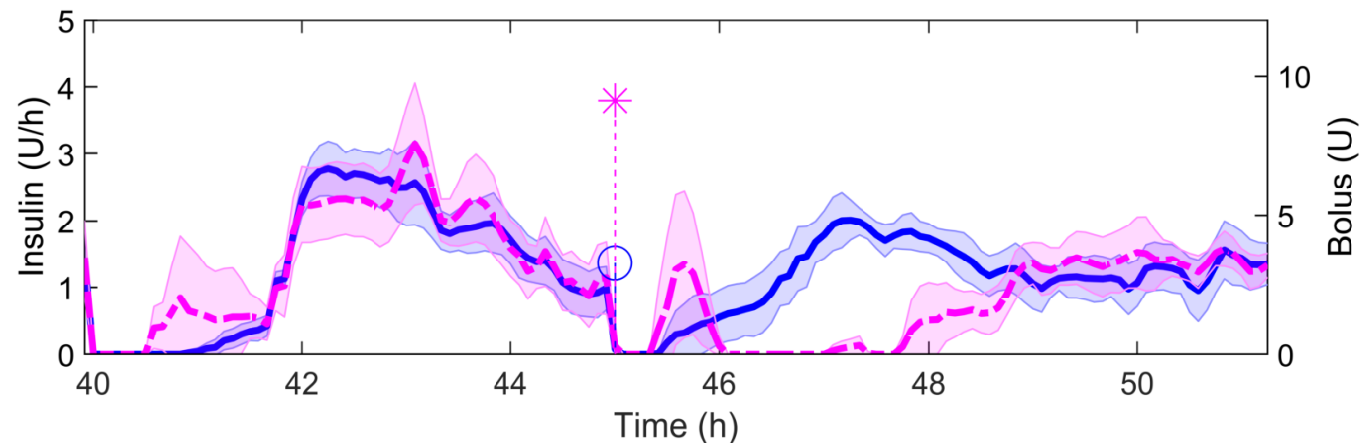

Figure 11. Population insulin trajectory (MEAN \pm STD) when an exercise announcement patient-inthe-loop fault affects the system. The blue curve represents the controller without reconfiguration and the magenta curve represents the controller with reconfiguration. The circular and star points at the 45 th simulation hour represent the mean boluses taken in each simulation.

\section{Discussion}

The patient-in-the-loop concept is fundamental for any diabetes treatment. Patients play essential roles within the treatment, from being the plant to control to actuate as an operator. This is the case for both OL and CL insulin therapies, and significantly more important in OL strategies where patients have more responsibility. Errors introduced by patients may compromise the performance and stability of treatments and patients may put themselves at risk. To minimize patient-in-the-loop faults, tools that monitor and detect those behaviors are needed. Detection of faults may allow CL controllers to take additional 
corrective actions to minimize the impact of the fault on the system performance. However, not all patient-in-the-loop faults might be easy to respond to. In any situation, monitoring systems will allow the collection of patient data that could be used to individualize the tuning of controllers or identify when faults are most likely to happen. Additionally, the detection of patient-in-the-loop faults with minimal impact during CL operation can be hard. CL systems are designed to reduce variability of the controlled variable and for disturbance rejection. Therefore, faults that have a small impact on the system and on BG concentration might be counteracted by the CL system itself and not detected as a fault. This is the case of misestimated meals in hybrid AP settings. In the case of OL therapies, this issue should be of less importance, allowing for more accurate detection.

To tackle this issue, we proposed a methodology based on a HA model. Even though the results we obtained were satisfactory and promising, our study suffered from several limitations. The first one is related to the data used to classify patient modes and faults. We used a simulation environment to stress the system and check its robustness and performance in several proof-of-concept examples. Certain limitations exist when trying to replicate real patient-in-the-loop faults in a simulation environment. BG trajectories may significantly vary depending on what the patient is performing, which may fall under normal operation. For example, postprandial periods where the patient is mostly in a sedentary state may differ from periods where the patient engages in slow walks. In any situation, these should not be detected as different patient states since there is no abnormality in the system. Real free-living condition data is still needed to assess the performance and detection capabilities of the proposed approach.

In this approach, we used a bank of interval zonotopic Kalman filters for the purpose of generating residuals. There are limitations to this approach due to the fact of using an approximate model and limited measurements. Particularly, the altered sensitivity observer has been designed to detect decreases in BG caused by aerobic exercise. Other types of exercise may have different effects on insulin sensitivity, requiring additional observers. Other factors such as illness, stress, menstruation cycle, sleep apnea, or some medications can also lead to important physiological changes, including changes in insulin sensitivity $[52,53]$. Therefore, the system is assumed to be working under normal operation for the detection of aerobic exercise. The major limitation of using a bank of observers is the need to build many different observers for the detection of the multitude of factors encountered in free living conditions. We used a reduced glucoregulatory model to relax the problem of observability of linear systems when only one measurement is available, but many states have to be estimated. Even though we only used the observers as a mean to generate residuals, the information from the observers could also be used to estimate other physiological parameters, such as the rates of glucose absorption.

We used the basic AP configuration, which only includes a CGM and an insulin pump. This was decided because even though there are studies using additional signals (such as using heart rate monitors, energy expenditure information and galvanic skin responses) these additional signals mean that the user (the patient) will have to wear additional sensors. Thus, although this could be of benefit for better detection performance, it also means that there is an increased risk of faults because more devices are used. Ultimately, the CGM and an insulin pump are always required devices for any AP operation. The proposed approach has two goals: (1) provide information for the AP in real time, and (2) provide information for long term decision making. By classifying patient errors, we believe that the system can be fine-tuned to maximize performance and minimize the risk of hypoglycemia due to patient actions. The developed approach could also work with different AP configurations, for example, AP systems with additional control signals such as glucagon [54]. This holds true because the developed approach is model-based, and by adding extra control actions the real model (the patient) retains the same dynamics. However, notice that this may require a re-tuning of the approach since not all possible modes have been considered, for example, faults regarding glucagon. The system performance may be compromised if used with other treatment options such as cadaveric islet or stem-cell derived islet 
transplantation. This also holds true when using the proposed approach for very specific sub-cohorts of people with T1D. All approaches that may modify the open-loop dynamics of the patient will require a model check for the proposed system to work.

Automated insulin delivery systems are still under research and improvement, with few systems such as the Medtronic $670 \mathrm{G}$ and newer $780 \mathrm{G}$ available on the market [55]. The most common AP uses the so-called subcutaneous route to sense glucose and deliver insulin. Non-physiological routes inevitably create non-physiological delays that are nonexistent in the healthy endogenous beta cells when sensing glucose and secreting insulin [56]. The major limiting factor of the subcutaneous route is related to the absorption of fast or ultra-fast insulin analogues. Most of these insulin formulations start actuating 10-15 min after their injection with a maximum peak of action between 1 and $2 \mathrm{~h}$ [57]. One of the consequences of this is the fact that most AP systems are rather conservative in insulin delivery to avoid overdoses of insulin, which may lead to hypoglycemia. As new insulins come to the market with faster profiles, the AP systems should be able to better control blood glucose due to a lowered delay of the control action effect. For example, newer formulations of insulin Aspart such as AT247, IAsp and FIAsp still have an approximate onset of exposure of $5 \mathrm{~min}$ [58-60]. On the other hand, CGM sensors have gradually improved their accuracy and some models can already be used as non-adjunctive, such as de Dexcom G6 [61]. Delays in blood glucose sensing by CGMs have been acknowledged for a long time [62]. Newer models already reported MARD $<10 \%$, however, delays between plasma insulin and measured CGM glucose concentrations are still in the range of 5-10 min [61]. CGM technology provides close to continuous flux of glucose readings, generally between 1 and $5 \mathrm{~min}$. Although this measurement sampling rate is much slower compared to how fast the healthy endogenous beta cells sense glucose, it is fast enough to control blood glucose since the dynamics of the overall system are much slower.

Current AP systems will certainly improve their performance if the delays associated with the subcutaneous route are reduced [56]. Especially, in situations where fast disturbances affect the system, for example for postprandial control [63]. Recent clinical trial outcomes of AP systems suggest that night periods are better controlled compared to traditional therapies [8], while day periods remain a challenge. This is caused by the absence of disturbances, mainly meals, during nights. Therefore, controlling postprandial periods is still difficult for AP systems even with the newest improved algorithms. Improving the absorption of insulin analogues will most likely allow for better control of postprandial periods than improving the already tested algorithms. The proposed approach should be able to accommodate improvements for newer insulin formulations and CGM systems since it is model-based. Theoretically, these improvements are equivalent to changing the time constants of the insulin and glucose sub-systems change. Then, the proposed approach would require including these new time constants in the models used. Clearly, the model change will have to be validated and the system performance assessed again.

Detection performance should be analyzed in depth before performing controller reconfiguration. While some patient-in-the-loop faults might be easier to detect with high accuracy, others might show higher rate of FP and FN. The results presented in this paper showed that detecting faults in $\mathrm{CHO}$ control actions can be accurately performed, while detecting errors in meal estimation is harder. The detection of meal misestimations is especially critical for the case of FP events, where controller reconfiguration could be triggered erroneously. This is something that must be taken into consideration when designing the FTC strategy. Detecting faults of people with type 1 diabetes is a complex task. Unfortunately, to discriminate different faults, the use of different observers is required. Nevertheless, the HA model is not restricted to work with observers. Residuals can be generated in a multitude of ways. In this work we used a bank of observers for the generation of residuals. However, any other methodology for the residual generation can be used and incorporated into the HA model. 


\title{
8. Conclusions
}

A methodology to monitor patient-in-the-loop modes and faults has been presented. The system is built around a HA model that replicate patient's real life operation modes. Transitions between different modes allow for the identification of key operational modes and faults. The diagnoser is responsible for mode recognition by using a group of indicators generated from a set of residuals for every mode. A bank of interval zonotopic Kalman filters was constructed for the residual generation, allowing the system to have confidence bounds on the state estimation and residual generation.

Several proof of concept simulated benchmarks were done using challenging scenarios. The results suggest that mode and patient-in-the-loop faults can be detected in real time. Next, the information resulting from this study could be used as a tool to reconfigure CL controllers, monitor the system continuously and classify patient-in-the-loop behaviors. The exploration of these areas will be part of future research. Moreover, the adaptation of the LPV Hovorka reduced model to represent glycemic dynamics will be investigated.

Author Contributions: Conceptualization, A.B. and V.P.; methodology, A.B., V.P., B.W.B. and J.V.; software, A.B.; validation, A.B.; formal analysis, A.B. and V.P.; investigation, A.B.; resources, V.P., B.W.B. and J.V.; data curation, A.B.; writing-original draft preparation, A.B.; writing-review and editing, V.P., B.W.B. and J.V.; visualization, A.B.; supervision, V.P., B.W.B. and J.V.; project administration, V.P. and J.V.; funding acquisition, J.V. All authors have read and agreed to the published version of the manuscript.

Funding: This work was partially supported by the Spanish Ministry of Science and Innovation through grant PID2019-107722RB-C22, in part by the Autonomous Government of Catalonia under Grant 2017 SGR 1551, in part by the Ministerio de Educación, Cultura y Deporte under Grant FPU0244 2015, and in part EU through FEDER funds.

Institutional Review Board Statement: Not applicable.

Informed Consent Statement: Not applicable.

Conflicts of Interest: The authors declare no conflict of interest.

\author{
Abbreviations \\ The following abbreviations are used in this manuscript: \\ T1D Type 1 Diabetes \\ BG Blood Glucose \\ AP Artificial Pancreas \\ CL Closed-Loop \\ CGM Continuous Glucose Monitoring \\ FTC Fault Tolerant Control \\ FD Fault Detection \\ FI Fault Identification \\ $\mathrm{CHO}$ Carbohydrates \\ LPV Linear Parameter Varying \\ LQC Linear Quadratic Control \\ LMI Linear Matrix Inequalities \\ IO Interval Observers \\ HA Hybrid Automaton \\ IFB Insulin Feedback \\ PD Proportional-Derivative \\ IOB Insulin-On-Board \\ SMRC Sliding Mode Reference Conditioning \\ COB Carbohydrates-On-Board \\ FDA Food and Drug Administration
}




\section{References}

1. Jacobson, A.M.; Braffett, B.H.; Cleary, P.A.; Gubitosi-Klug, R.A.; Larkin, M.E.; DCCT/EDIC Research Group. The long-term effects of type 1 diabetes treatment and complications on health-related quality of life: A 23-year follow-up of the Diabetes Control and Complications/Epidemiology of Diabetes Interventions and Complications cohort. Diabetes Care 2013, 36, $3131-3138$. [CrossRef] [PubMed]

2. Cade, W.T. Diabetes-related microvascular and macrovascular diseases in the physical therapy setting. Phys. Ther. 2008, 88, 1322-1335. [CrossRef] [PubMed]

3. Walsh, J. Using Insulin: Everything You Need for Success with Insulin; Torrey Pines Press: San Diego, CA, USA, 2003.

4. Walsh, J.; Roberts, R. Pumping Insulin: Everything You Need for Success on a Smart Insulin Pump; Torrey Pines Press: San Diego, CA, USA, 2003.

5. Doyle, F.J.; Huyett, L.M.; Lee, J.B.; Zisser, H.C.; Dassau, E. Closed-loop artificial pancreas systems: Engineering the algorithms. Diabetes Care 2014, 37, 1191-1197. [CrossRef] [PubMed]

6. Thabit, H.; Hovorka, R. Coming of age: The artificial pancreas for type 1 diabetes. Diabetologia 2016, 59, 1795-1805. [CrossRef]

7. Bertachi, A.; Ramkissoon, C.M.; Bondia, J.; Vehí, J. Automated blood glucose control in type 1 diabetes: A review of progress and challenges. Endocrinol. Diabetes Nutr. 2018, 65, 172-181. [CrossRef]

8. Bekiari, E.; Kitsios, K.; Thabit, H.; Tauschmann, M.; Athanasiadou, E.; Karagiannis, T.; Haidich, A.-B.; Hovorka, R.; Tsapas, A. Artificial pancreas treatment for outpatients with type 1 diabetes: Systematic review and meta-analysis. BMJ 2018, $361, \mathrm{k} 1310$. [CrossRef]

9. Ramkissoon, C.M.; Aufderheide, B.; Bequette, B.W.; Vehí, J. A review of safety and hazards associated with the artificial pancreas. IEEE Rev. Biomed. Eng. 2017, 10, 44-62. [CrossRef] [PubMed]

10. Bequette, B.W. Human-in-the-loop insulin dosing. J. Diabetes Sci. Technol. 2021, 15, 699-704. [CrossRef] [PubMed]

11. Saunders, A.; Messer, L.H.; Forlenza, G.P. MiniMed 670G hybrid closed loop artificial pancreas system for the treatment of type 1 diabetes mellitus: Overview of its safety and efficacy. Expert Rev. Med. Devices 2019, 16, 845-853. [CrossRef]

12. Turksoy, K.; Roy, A.; Cinar, A. Real-time model-based fault detection of continuous glucose sensor measurements. IEEE Trans. Biomed. Eng. 2016, 64, 1437-1445. [CrossRef] [PubMed]

13. Mahmoudi, Z.; Nørgaard, K.; Poulsen, N.K.; Madsen, H.; Jørgensen, J.B. Fault and meal detection by redundant continuous glucose monitors and the unscented Kalman filter. Biomed. Signal Process. Control 2017, 38, 86-99. [CrossRef]

14. Howsmon, D.P.; Cameron, F.; Baysal, N.; Ly, T.T.; Forlenza, G.P.; Maahs, D.M.L.; Buckingham, B.A.; Hahn, J.; Bequette, B.W. Continuous glucose monitoring enables the detection of losses in infusion set actuation (LISAs). Sensors 2017, 17, 161. [CrossRef] [PubMed]

15. Meneghetti, L.; Terzi, M.; Del Favero, S.; Susto, G.A.; Cobelli, C. Data-driven anomaly recognition for unsupervised model-free fault detection in artificial pancreas. IEEE Trans. Control. Syst. Technol. 2018, 28, 33-47. [CrossRef]

16. Meneghetti, L.; Susto, G.A.; Del Favero, S. Detection of insulin pump malfunctioning to improve safety in artificial pancreas using unsupervised algorithms. J. Diabetes Sci. Technol. 2019, 13, 1065-1076. [CrossRef] [PubMed]

17. Baysal, N.; Cameron, F.; Buckingham, B.A.; Wilson, D.M.; Chase, H.P.; Maahs, D.M.; In Home Closed-Loop Study Group (IHCL). A novel method to detect pressure-induced sensor attenuations (PISA) in an artificial pancreas. J. Diabetes Sci. Technol. 2014, 8, 1091-1096. [CrossRef] [PubMed]

18. Samadi, S.; Rashid, M.; Turksoy, K.; Feng, J.; Hajizadeh, I.; Hobbs, N.; Cinar, A. Automatic detection and estimation of unannounced meals for multivariable artificial pancreas system. Diabetes Technol. Ther. 2018, 20, 235-246. [CrossRef] [PubMed]

19. Ramkissoon, C.M.; Herrero, P.; Bondia, J.; Vehi, J. Unannounced meals in the artificial pancreas: Detection using continuous glucose monitoring. Sensors 2018, 18, 884. [CrossRef]

20. Sala-Mira, I.; Diez, J.L.; Ricarte, B.; Bondia, J. Sliding-mode disturbance observers for an artificial pancreas without meal announcement. J. Process. Control. 2019, 78, 68-77. [CrossRef]

21. Sanz, R.; García, P.; Díez, J.L.; Bondia, J. Artificial Pancreas System with Unannounced Meals Based on a Disturbance Observer and Feedforward Compensation. IEEE Trans. Control. Syst. Technol. 2020, 29, 454-460. [CrossRef]

22. Cameron, F.; Niemeyer, G.; Wilson, D.M.; Bequette, B.W.; Benassi, K.S.; Clinton, P.; Buckingham, B.A. Inpatient trial of an artificial pancreas based on multiple model probabilistic predictive control with repeated large unannounced meals. Diabetes Technol. Ther. 2014, 16, 728-734. [CrossRef]

23. Cameron, F.M.; Ly, T.T.; Buckingham, B.A.; Maahs, D.M.; Forlenza, G.P.; Levy, C.J.; Levister, C. Closed-loop control without meal announcement in type 1 diabetes. Diabetes Technol. Ther. 2017, 19, 527-532. [CrossRef] [PubMed]

24. Jacobs, P.G.; Resalat, N.; El Youssef, J.; Reddy, R.; Branigan, D.; Preiser, N.; Castle, J. Incorporating an exercise detection, grading, and hormone dosing algorithm into the artificial pancreas using accelerometry and heart rate. J. Diabetes Sci. Technol. 2015, 9 , 1175-1184. [CrossRef] [PubMed]

25. Ramkissoon, C.M.; Bertachi, A.; Beneyto, A.; Bondia, J.; Vehi, J. Detection and control of unannounced exercise in the artificial pancreas without additional physiological signals. IEEE J. Biomed. Health Inform. 2019, 24, 259-267. [CrossRef]

26. Beneyto, A.; Bequette, B.W.; Vehi, J. Fault Tolerant Strategies for Automated Insulin Delivery Considering the Human Component: Current and Future Perspectives. J. Diabetes Sci. Technol. 2021. [CrossRef] [PubMed]

27. Beneyto, A.; Bertachi, A.; Bondia, J.; Vehi, J. A new blood glucose control scheme for unannounced exercise in type 1 diabetic subjects. IEEE Trans. Control. Syst. Technol. 2018, 28, 593-600. [CrossRef] 
28. Brazeau, A.S.; Mircescu, H.; Desjardins, K.; Leroux, C.; Strychar, I.; Ekoé, J.M.; Rabasa-Lhoret, R. Carbohydrate counting accuracy and blood glucose variability in adults with type 1 diabetes. Diabetes Res. Clin. Pract. 2013, 99, 19-23. [CrossRef] [PubMed]

29. Mohammadpour, J.; Scherer, C.W. (Eds.) Control of Linear Parameter Varying Systems with Applications; Springer Science \& Business: New York, NY, USA, 2012.

30. Revert, A.; Garelli, F.; Picó, J.; De Battista, H.; Rossetti, P.; Vehí, J.; Bondia, J. Safety auxiliary feedback element for the artificial pancreas in type 1 diabetes. IEEE Trans. Biomed. Eng. 2013, 60, 2113-2122. [CrossRef]

31. León-Vargas, F.; Garelli, F.; De Battista, H.; Vehí, J. Postprandial response improvement via safety layer in closed-loop blood glucose controllers. Biomed. Signal Process. Control. 2015, 16, 80-87. [CrossRef]

32. Bertachi, A.; Beneyto, A.; Ramkissoon, C.M.; Vehi, J. Assessment of mitigation methods to reduce the risk of hypoglycemia for announced exercise in a uni-hormonal artificial pancreas. Diabetes Technol. Ther. 2018, 20, 285-295. [CrossRef]

33. Bertachi, A.; Biagi, L.; Beneyto, A.; Vehí, J. Dynamic rule-based algorithm to tune insulin-on-board constraints for a hybrid artificial pancreas system. J. Healthc. Eng. 2020, 2020, 1414597. [CrossRef] [PubMed]

34. Rossetti, P.; Quiros, C.; Moscardo, V.; Comas, A.; Giménez, M.; Ampudia-Blasco, F.J.; Vehí, J. Closed-loop control of postprandial glycemia using an insulin-on-board limitation through continuous action on glucose target. Diabetes Technol. Ther. 2017, 19, 355-362. [CrossRef]

35. Quirós, C.; Bertachi, A.; Giménez, M.; Biagi, L.; Viaplana, J.; Viñals, C.; Bondia, J. Control de la glucemia durante el ejercicio físico aeróbico y anaeróbico mediante un nuevo sistema de páncreas artificial. Endocrinol. Diabetes Nutr. 2018, 65, 342-347. [CrossRef]

36. Viñals, C.; Beneyto, A.; Martín-SanJosé, J.F.; Furió-Novejarque, C.; Bertachi, A.; Bondia, J.; Giménez, M. Artificial pancreas with carbohydrate suggestion performance for unannounced and announced exercise in Type 1 Diabetes. J. Clin. Endocrinol. Metab. 2021, 106, 55-63. [CrossRef]

37. Vento, J.; Travé-Massuyès, L.; Puig, V.; Sarrate, R. An incremental hybrid system diagnoser automaton enhanced by discernibility properties. IEEE Trans. Syst. Man Cybern. Syst. 2014, 45, 788-804. [CrossRef]

38. Bergman, R.N.; Ider, Y.Z.; Bowden, C.R.; Cobelli, C. Quantitative estimation of insulin sensitivity. Am. J. -Physiol.-Endocrinol. Metab. 1979, 236, E667. [CrossRef] [PubMed]

39. Visentin, R.; Campos-Náñez, E.; Schiavon, M.; Lv, D.; Vettoretti, M.; Breton, M.; Cobelli, C. The UVA/Padova type 1 diabetes simulator goes from single meal to single day. J. Diabetes Sci. Technol. 2018, 12, 273-281. [CrossRef]

40. Hovorka, R.; Canonico, V.; Chassin, L.J.; Haueter, U.; Massi-Benedetti, M.; Federici, M.O.; Wilinska, M.E. Nonlinear model predictive control of glucose concentration in subjects with type 1 diabetes. Physiol. Meas. 2004, 25, 905. [CrossRef]

41. Bondia, J.; Romero-Vivo, S.; Ricarte, B.; Diez, J.L. Insulin estimation and prediction: A review of the estimation and prediction of subcutaneous insulin pharmacokinetics in closed-loop glucose control. IEEE Control. Syst. Mag. 2018, 38, 47-66.

42. Kovács, L.; Szalay, P. Uncertainties and Modeling Errors of Type 1 Diabetes Models. In Prediction Methods for Blood Glucose Concentration; Springer: Cham, Switzerland, 2016; pp. 211-225.

43. Kwiatkowski, A.; Boll, M.T.; Werner, H. Automated generation and assessment of affine LPV models. In Proceedings of the 45th IEEE Conference on Decision and Control, San Diego, CA, USA, 13-15 December 2006; pp. 6690-6695.

44. Sun, X.D.; Postlethwaite, I. Affine LPV modelling and its use in gain-scheduled helicopter control. In Proceedings of the UKACC International Conference on Control'98, Swansea, UK, 1-4 September 1998; pp. 1504-1509.

45. Rotondo, D.; Nejjari, F.; Puig, V. Quasi-LPV modeling, identification and control of a twin rotor MIMO system. Control. Eng. Pract. 2013, 21, 829-846. [CrossRef]

46. Alcala, E.; Puig, V.; Quevedo, J.; Escobet, T. Gain-scheduling LPV control for autonomous vehicles including friction force estimation and compensation mechanism. IET Control. Theory Appl. 2018, 12, 1683-1693. [CrossRef]

47. Ostertag, E. Mono- and Multivariable Control and Estimation: Linear, Quadratic and LMI Methods; Springer Science \& Business: Berlin/Heidelberg, Germany, 2011.

48. Xu, F.; Puig, V.; Ocampo-Martinez, C.; Olaru, S.; Stoican, F. Set-theoretic methods in robust detection and isolation of sensor faults. Int. J. Syst. Sci. 2015, 46, 2317-2334. [CrossRef]

49. Man, C.D.; Micheletto, F.; Lv, D.; Breton, M.; Kovatchev, B.; Cobelli, C. The UVA/PADOVA type 1 diabetes simulator: New features. J. Diabetes Sci. Technol. 2014, 8, 26-34. [CrossRef]

50. Herrero, P.; Bondia, J.; Oliver, N.; Georgiou, P. A coordinated control strategy for insulin and glucagon delivery in type 1 diabetes. Comput. Methods Biomech. Biomed. Eng. 2017, 20, 1474-1482. [CrossRef]

51. Dalla Man, C.; Breton, M.D.; Cobelli, C. Physical Activity into the Meal Glucose-Insulin Model of Type 1 Diabetes: In Silico Studies. J. Diabetes Sci. Technol. 2009, 3, 56-67. [CrossRef]

52. Pulido, J.M.E.; Salazar, M.A. Changes in insulin sensitivity, secretion and glucose effectiveness during menstrual cycle. Arch. Med. Res. 1999, 30, 19-22. [CrossRef]

53. Pretty, C.; Le Compte, A.; Chase, J.G.; Shaw, G.; Preiser, J.C.; Penning, S.; Desaive, T. Variability of insulin sensitivity during the first 4 days of critical illness. Crit. Care 2012, 16, 1-189. [CrossRef]

54. Moscardó, V.; Díez, J.L.; Bondia, J. Parallel control of an artificial pancreas with coordinated insulin, glucagon, and rescue carbohydrate control actions. J. Diabetes Sci. Technol. 2019, 13, 1026-1034. [CrossRef]

55. Allen, N.; Gupta, A. Current diabetes technology: Striving for the artificial pancreas. Diagnostics 2019, 9, 31. [CrossRef] [PubMed]

56. Christiansen, S.C.; Fougner, A.L.; Stavdahl, Ø.; Kölle, K.; Ellingsen, R.; Carlsen, S.M. A review of the current challenges associated with the development of an artificial pancreas by a double subcutaneous approach. Diabetes Ther. 2017, 8, 489-506. [CrossRef] 
57. Hirsch, I.B.; Juneja, R.; Beals, J.M.; Antalis, C.J.; Wright, E.E., Jr. The evolution of insulin and how it informs therapy and treatment choices. Endocr. Rev. 2020, 41, 733-755. [CrossRef] [PubMed]

58. Haahr, H.; Heise, T. Fast-acting insulin aspart: A review of its pharmacokinetic and pharmacodynamic properties and the clinical consequences. Clin. Pharmacokinet. 2020, 59, 155-172. [CrossRef] [PubMed]

59. Heise, T.; Hövelmann, U.; Brøndsted, L.; Adrian, C.L.; Nosek, L.; Haahr, H. Faster-acting insulin aspart: Earlier onset of appearance and greater early pharmacokinetic and pharmacodynamic effects than insulin aspart. Diabetes Obes. Metab. 2015, 17, 682-688. [CrossRef] [PubMed]

60. Svehlikova, E.; Mursic, I.; Augustin, T.; Magnes, C.; Gerring, D.; Jezek, J.; Pieber, T.R. Pharmacokinetics and Pharmacodynamics of Three Different Formulations of Insulin Aspart: A Randomized, Double-Blind, Crossover Study in Men with Type 1 Diabetes. Diabetes Care 2021, 44, 448-455. [CrossRef]

61. Cappon, G.; Vettoretti, M.; Sparacino, G.; Facchinetti, A. Continuous glucose monitoring sensors for diabetes management: A review of technologies and applications. Diabetes Metab. J. 2019, 43, 383-397. [CrossRef]

62. Schmelzeisen-Redeker, G.; Schoemaker, M.; Kirchsteiger, H.; Freckmann, G.; Heinemann, L.; Del Re, L. Time delay of CGM sensors: Relevance, causes, and countermeasures. J. Diabetes Sci. Technol. 2015, 9, 1006-1015. [CrossRef] [PubMed]

63. Goodwin, G.C.; Medioli, A.M.; Carrasco, D.S.; King, B.R.; Fu, Y. A fundamental control limitation for linear positive systems with application to Type 1 diabetes treatment. Automatica 2015, 55, 73-77. [CrossRef] 